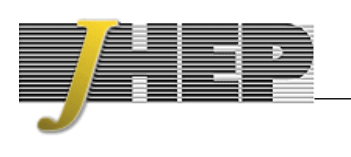

\title{
Search for natural supersymmetry in events with top quark pairs and photons in pp collisions at $\sqrt{s}=8 \mathrm{TeV}$
}

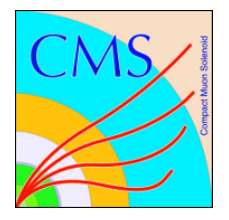

\section{The CMS collaboration}

\section{E-mail: cms-publication-committee-chair@cern.ch}

ABSTRACT: Results are presented from a search for natural gauge-mediated supersymmetry (SUSY) in a scenario in which the top squark is the lightest squark, the next-to-lightest SUSY particle is a bino-like neutralino, and the lightest SUSY particle is the gravitino. The strong production of top squark pairs can produce events with pairs of top quarks and neutralinos, with each bino-like neutralino decaying to a photon and a gravitino. The search is performed using a sample of pp collision data accumulated by the CMS experiment at $\sqrt{s}=8 \mathrm{TeV}$, corresponding to an integrated luminosity of $19.7 \mathrm{fb}^{-1}$. The final state consists of a lepton (electron or muon), jets, and one or two photons. The imbalance in transverse momentum in the events is compared with the expected spectrum from standard model processes. No excess event yield is observed beyond the expected background, and the result is interpreted in the context of a general model of gauge-mediated SUSY breaking that leads to exclusion of top squark masses below $650-730 \mathrm{GeV}$.

KEYWORDS: Hadron-Hadron scattering (experiments), Supersymmetry

ArXiv EPRINT: 1707.03325 


\section{Contents}

1 Introduction 1

2 The CMS detector 2

3 Object reconstruction 3

4 Event selection and analysis strategy 4

$\begin{array}{llr}5 & \text { Results and interpretation } & 9\end{array}$

$\begin{array}{lll}6 & \text { Summary } & 13\end{array}$

$\begin{array}{lr}\text { The CMS collaboration } & 19\end{array}$

\section{Introduction}

Supersymmetry (SUSY) [1-9] is an extension of the standard model (SM) that can avoid the hierarchy [10-15] problem confirmed by the recent discovery $[16,17]$ of the Higgs boson at a mass of $125 \mathrm{GeV}$ [18]. Of particular interest are the "natural" models of SUSY in which several SUSY partners (sparticles) of SM particles are expected to be light, such as the third-generation squarks and the Higgsinos [19, 20]. In such SUSY scenarios, the fine-tuning required to maintain low mass of the electroweak scale can be avoided. Many light top squark searches are being pursued at the LHC [21-26].

In this paper, we describe a search for light top squarks $(\widetilde{\mathrm{t}})$ in a data sample corresponding to an integrated luminosity of $19.7 \mathrm{fb}^{-1}$ of pp collisions at $\sqrt{s}=8 \mathrm{TeV}$. This search is motivated by models of gauge-mediated SUSY breaking (GMSB) [27-29] in which the neutralino $\left(\widetilde{\chi}_{1}^{0}\right)$ is the next-to-lightest sparticle (NLSP) and the gravitino $(\tilde{G})$ is the lightest sparticle (LSP). The gravitino escapes undetected and contributes to missing transverse momentum $\left(\vec{p}_{\mathrm{T}}^{\text {miss }}\right)$ in the detector, where the magnitude of $\vec{p}_{\mathrm{T}}^{\text {miss }}$ is referred to as $p_{\mathrm{T}}^{\text {miss }}$. This search considers a bino-like neutralino that decays to a photon and a gravitino. Assuming that $R$-parity $[30,31]$ is conserved, pair production of sparticles would be the dominant production mechanism for SUSY particles in pp collisions at the LHC. Because top squarks are expected to be relatively light in natural SUSY scenarios, we search for top squark pair production, a strong process. Assuming a bino-like neutralino NLSP, each top squark would decay to a top quark and a neutralino, with the neutralino decaying to a photon and a gravitino, leading to a $\mathrm{t} \overline{\mathrm{t}}+\gamma \gamma+p_{\mathrm{T}}^{\text {miss }}$ topology. This event topology is shown in figure 1.

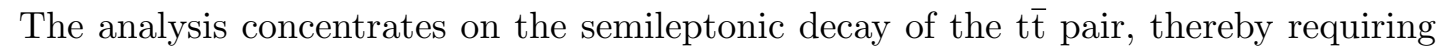
the presence of exactly one isolated electron or muon. This minimizes contributions from 


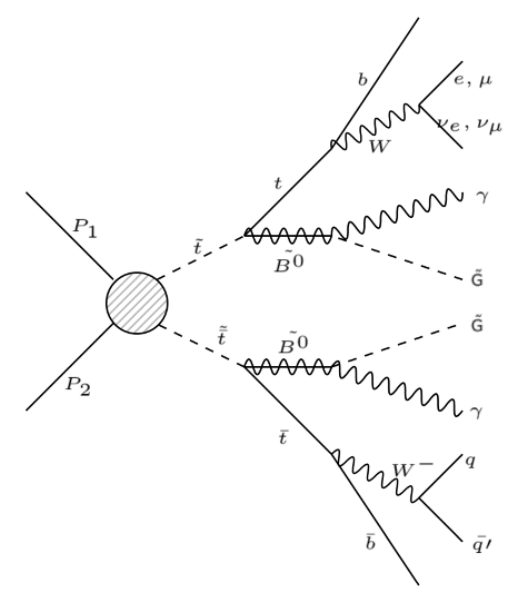

Figure 1. The event topology used to search for low mass top squarks pairs.

multijet and $\gamma+$ jet backgrounds. At least one jet in each event is required to be tagged as originating from a $b$ quark to reduce non-t $\bar{t}$ backgrounds. No explicit $t \bar{t}+\gamma \gamma$ sample is used in the background estimates because of the exceedingly small cross section for such events in the SM. Two signal regions are defined for both electron and muon channels, depending on the presence of one or two selected photons in the event. Control regions are similarly defined, using photons that fail either the nominal isolation or shower-energy distribution requirements.

The results of the analysis are evaluated by comparing the shapes of $p_{\mathrm{T}}^{\text {miss }}$ distributions between the data and estimated backgrounds in the one- and two-photon signal regions. The results are interpreted for a range of top squark and neutralino masses in a general gauge-mediated (GGM) SUSY model framework [32-37].

\section{The CMS detector}

The central feature of the CMS detector is a superconducting solenoid with an internal diameter of $6 \mathrm{~m}$, providing a magnetic field of $3.8 \mathrm{~T}$. A silicon pixel and strip tracker, a lead tungstate crystal electromagnetic calorimeter (ECAL), and a brass and scintillator sampling hadron calorimeter (HCAL), each separated into central barrel and endcap sections, reside within the field volume. Extensive forward calorimetry complements the coverage provided by the barrel and endcap detectors. The muon system, embedded in the steel return yoke outside of the solenoid, measures muons using drift tubes, cathode strip chambers, and resistive plate chambers.

An energy resolution averaging approximately $1 \%$ is achieved for unconverted or lateconverting photons in the energy range of photons in the barrel section of ECAL. The remaining converted barrel photons have a resolution of about $1.3 \%$ up to a pseudorapidity of $|\eta|=1$, rising to about $2.5 \%$ at $|\eta|=1.4$ [38]. Only photons located in the barrel of the ECAL are considered in this analysis because of the superior energy resolution in the barrel compared to the endcap. 
The first level of the CMS trigger system, constructed using special hardware processors, provides information from the calorimeters and muon detectors to select the most interesting events in a fixed time interval of less than $4 \mu \mathrm{s}$. The high-level trigger processor farm further decreases the event rate from around $100 \mathrm{kHz}$ to about $400 \mathrm{~Hz}$ before data storage.

A more detailed description of the CMS detector, together with a definition of the coordinate system and the kinematic variables, such as $\eta$ or the azimuthal angle $\phi$ (in radians), can be found in ref. [39].

\section{Object reconstruction}

All physics objects in the event (muons, electrons, photons, jets, and $p_{\mathrm{T}}^{\mathrm{miss}}$ ) are reconstructed using the particle-flow (PF) algorithm [40, 41]. Jets are formed by clustering PF candidates using the anti- $k_{\mathrm{T}}$ algorithm [42], as implemented in FASTJET toolkit [43], using a distance parameter of 0.5 , and their momenta are corrected for effects of multiple interactions in the same or neighboring bunch crossings (pileup). The $p_{\mathrm{T}}^{\text {miss }}$ of an event is defined by the projection of the negative of the vector sum of the momenta of all reconstructed objects in the event onto the plane perpendicular to the proton beams. All PF candidates are used in the calculation of $p_{\mathrm{T}}^{\mathrm{miss}}$.

Photons are reconstructed from energy clusters in the ECAL barrel $(|\eta|<1.44)$, are required to be highly isolated from other objects, and to have transverse momentum $p_{\mathrm{T}}>$ $20 \mathrm{GeV}$. The ratio of the energy deposited in the HCAL tower closest to the seed of the ECAL photon cluster to the energy in the photon cluster has to be less than five percent. The photon shower is required to have a photon-like spatial distribution in its energy [38]. The isolation variable, defined through the sum of the scalar values of $p_{\mathrm{T}}$ of all PF candidates within a cone centered on the photon axis, in the $\eta-\phi$ plane of $\Delta R=$ $\sqrt{(\Delta \phi)^{2}+(\Delta \eta)^{2}}=0.3$, is calculated without including the $p_{\mathrm{T}}$ of the candidate photon. The isolation energy for charged hadrons is required to be $<15 \mathrm{GeV}$, the neutral-hadron energy $<3.5 \mathrm{GeV}+4 \%$ of the photon candidate $p_{\mathrm{T}}$, and the isolation energy from any other photons in the cone must be $<13 \mathrm{GeV}+0.5 \%$ of the candidate photon $p_{\mathrm{T}}$. Pileup corrections depending on $\eta$ are applied to all calculated isolation variables.

Electrons are reconstructed from clusters of deposited energy in the ECAL that are matched to a track in the silicon tracker [44]. Candidate electrons are required to have $p_{\mathrm{T}}>30 \mathrm{GeV}$, and to be within $|\eta|<2.5$, excluding the small transition region $(1.44<|\eta|<$ 1.52) between the ECAL barrel and the endcaps. Electrons are required to be isolated, with the sum of the energy deposition within a cone of radius $\Delta R=0.3$, excluding the electron, to correspond to $<10 \%$ of the momentum of the candidate electron.

Muons are reconstructed from measurements in the muon system and compatible track segments in the silicon tracker [45]. Candidate muons are required to have $p_{\mathrm{T}}>30 \mathrm{GeV}$, be within $|\eta|<2.1$, and to have an isolation energy sum in a cone of radius $\Delta R=0.4$, excluding the muon, of $<12 \%$ of their $p_{\mathrm{T}}$. Looser lepton requirements are applied to identify extra leptons that are used to veto the dilepton t $\bar{t}$ final states, as described in section 4 . 
The combined secondary vertex algorithm (CSV) $[46,47]$ is used to identify jets from b quarks. The CSV algorithm uses secondary vertices and track impact parameters to provide a discriminant separating b quark jets from charm, light quark, or gluon jets. The selection efficiency is about $70 \%$ for b quark jets and $20 \%$ for c quark jets. The rejection factor for lighter quark or gluon jets at this working point is about $2 \%$.

\section{Event selection and analysis strategy}

Events are required to pass either a single-electron or single-muon trigger, requiring one isolated electron or muon with minimum $p_{\mathrm{T}}$ of 27 or $24 \mathrm{GeV}$, respectively. In addition, the single-muon trigger requires the muon candidate to be within $|\eta|<2.1$. The trigger efficiency is approximately $100 \%$ using offline cuts on $p_{\mathrm{T}}$ of $30 \mathrm{GeV}$.

Only one lepton and at least three jets with $p_{\mathrm{T}}>30 \mathrm{GeV}$ and $|\eta|<2.4$ are required, with at least one of the three jets tagged as originating from a b quark. All objects are required to be separated from each other by at least $\Delta R=0.5$. Events containing additional leptons satisfying less restrictive criteria of $p_{\mathrm{T}}>10 \mathrm{GeV},|\eta|<2.5$, and isolation-energy sums with $<20 \%$ of their $p_{\mathrm{T}}$, are rejected.

After this preselection, events are separated into independent samples based on the number of candidate photons. Candidate photons are required to be separated from all jets by $\Delta R>0.7$. Two signal regions are defined, with SR1 containing one photon candidate, and SR2 at least two photon candidates.

Photons that fail either the shower-energy distribution or charged-hadron isolation criteria are referred to as fake photons. These objects are predominantly jets with large electromagnetic fluctuations in their hadronization and are used to define two control regions: CR1, containing one fake and no properly reconstructed photons, and CR2, containing two or more fake and no properly reconstructed photons. The control regions are defined not to overlap with signal regions, to have very small acceptance for signal, and to greatly enhance the population of photon-like jets that contribute most of the estimated background in signal regions. The control regions also provide events that can be used to study the performance of the $p_{\mathrm{T}}^{\text {miss }}$ simulation for poorly reconstructed photon-like objects in the signal region. The effect on the $p_{\mathrm{T}}^{\text {miss }}$ resolution from these poorly reconstructed photon-like objects is found to be negligible compared to the effect of $p_{\mathrm{T}}$ resolutions in the jets from the $t \bar{t}$ decays.

The background expected in the signal regions is largely dominated by $t \bar{t}+$ jets and $t \bar{t}+\gamma$ events, where many selected photons may originate from misreconstructed jets. These two processes are simulated in Monte Carlo (MC) using the leading-order (LO) MAdGRAPH 5.1.3 [48] matrix element generator matched to PYTHIA 6.426 [49] for parton showering and fragmentation. Simulated $t \bar{t}+\gamma$ events are generated in a $2 \rightarrow 7$ configuration $(\mathrm{pp} \rightarrow \mathrm{bbjj} \ell \nu \gamma)$. Approximately $0.6 \%$ of the simulated $t \bar{t}+$ jets events that contain a generator-level photon fall into the phase space of the $t \bar{t}+\gamma$ sample, and are removed to avoid double counting these events. Most other backgrounds are simulated with MADGraPH and matched to PYTHIA, including $\mathrm{W}+$ jets or $\mathrm{Z}+$ jets, $\mathrm{t} \overline{\mathrm{t}}+\mathrm{W}$ or $\mathrm{t} \overline{\mathrm{t}}+\mathrm{Z}, \mathrm{W}+\gamma$ or $\mathrm{Z}+\gamma$, and diboson $(\mathrm{ZZ}, \mathrm{WZ}$, and $\mathrm{WW})$ processes. Single top quark events are generated 


\begin{tabular}{|lcc|}
\hline Software Package & Purpose & Physics Processes \\
\hline MADGRAPH 5.1.3 & (LO) Matrix element generator & All backgrounds except single top \\
POWHEG 1.0 & (NLO) Matrix element generator & Single top quark backgrounds \\
PYTHIA 6.42 & Parton showering and fragmentation & All backgrounds and signal \\
TAUOLA & Decay of $\tau$ leptons & Single top quark backgrounds \\
Z2* tune & Modeling of underlying event & All backgrounds and signal \\
GEANT4 & Modeling of the CMS detector & All backgrounds and signal \\
CTEQ6M & Parton distribution functions (PDF) & All backgrounds and signal \\
SUSPECT 2.41 & Generation of GGM signal spectrum & Signal \\
PROSPINO 2.1 & (NLO) Cross section calculation & Signal \\
SDECAY 1.2 & Decay tables for GGM particles & Signal \\
\hline
\end{tabular}

Table 1. Software used in MC simulations of backgrounds.

with the next-to-lowest-order (NLO) generator POWHEG 1.0 [50], modeling the decay of $\tau$ leptons with tauola [51]. The $\mathrm{Z}^{*}$ tune $[52,53]$ is used for the underlying event. All simulated backgrounds are processed using the full simulation of the response of the CMS detector using the GEANT4 [54] package, and reconstructed under the same conditions as the data. These backgrounds are then normalized to the integrated luminosity of the data using their respective cross sections calculated at least at NLO. The CTEQ6M parton distribution functions (PDF) are used in the signal and background simulations [55]. A summary of the software used in the MC simulations of backgrounds is given in table 1.

In the muon + jets channel, the background from $\mathrm{Z}+$ jets and $\mathrm{Z}+\gamma$ events is very small because of the low probablility for a muon to be misidentified as a photon. In the electron + jets channel, however, these processes contribute more to the background, especially at low $p_{\mathrm{T}}^{\text {miss }}$, because the probability for an electron to be misidentified as a photon is much greater. This electron misidentification rate can be determined from the size of the peak at the $\mathrm{Z}$ boson mass in the invariant mass distribution of electron-photon pairs in the electron+jets channel of SR1. This rate depends on an estimate of the number of selected $\mathrm{Z}$ bosons in the electron+jets channel, the accuracy of which can be improved through the implementation of a scale factor $(S F)$ extracted to normalize the $\mathrm{Z}+$ jets and $\mathrm{Z}+\gamma \mathrm{MC}$ events in both the electron and muon channels. The $S F$ is measured imposing a dilepton selection similar to the one used in the SR1 selection, but altered to require two same-flavor leptons rather than just a single lepton. Events with additional leptons are vetoed, and no photons are required. A fit to the invariant mass of the dilepton system in data, using the $\mathrm{Z}+$ jets and $\mathrm{Z}+\gamma \mathrm{MC}$ events as the signal template and all other MC events as background templates, provides a normalization scale factor for both the $\mathrm{Z}+$ jets and $\mathrm{Z}+\gamma \mathrm{MC}$ events, labeled $S F_{\mathrm{Z}(\gamma)}$, in the electron and the muon channels.

Once this first $S F$ is applied to correct the MC estimate of the number of $\mathrm{Z}$ bosons, the $\mathrm{Z}$ resonance in the $\mathrm{SR} 1$ electron+jets channel is used to obtain a second scale factor $S F_{\mathrm{e} \rightarrow \gamma}$ which corrects the misidentification of electrons as photons. A fit to the invariant mass of the electron-photon system in SR1 data, with $p_{\mathrm{T}}^{\text {miss }}<50 \mathrm{GeV}$, to limit the presence of signal, is performed using the $\mathrm{Z}+$ jets and $\mathrm{Z}+\gamma \mathrm{MC}$ events to determine their contributions. Generator-level matching of reconstructed photons to generated electrons is applied to 


\begin{tabular}{|lcc|}
\hline Channel & $S F_{\mathrm{Z}(\gamma)}$ & $S F_{\mathrm{e} \rightarrow \gamma}$ \\
\hline $\mathrm{e}$ & $1.38 \pm 0.02 \pm 0.15$ & $1.58 \pm 0.03 \pm 0.04$ \\
$\mu$ & $1.60 \pm 0.02 \pm 0.17$ & - \\
\hline
\end{tabular}

Table 2. Measured values of scale factors, $S F_{\mathrm{Z}(\gamma)}$ and $S F_{\mathrm{e} \rightarrow \gamma}$, used to correct the MC predictions for $\mathrm{Z}+$ jets and $\mathrm{Z}+\gamma$ backgrounds and electron-to-photon misidentification. For the electron+jets channel, the product of the two is applied to $\mathrm{Z}+$ jets and $\mathrm{Z}+\gamma$ backgrounds. In the muon+jets channel, only the $S F_{\mathrm{Z}(\gamma)}$ scale factor is relevant. The first uncertainties are statistical, obtained from uncertainties in the resultant fits. The second uncertainties correspond to differences in the resulting scale factors, added in quadrature, that were obtained by allowing each systematic uncertainty to fluctuate up and down by one standard deviation and refitting.

increase the purity of the misidentified e $\gamma$ mass template. To increase the statistics available for each template, the $\mathrm{b}$ tagging requirement is removed from the $\mathrm{MC}$ events and from the data sample, as the misidentification does not depend on the presence of a b jet. From the result of this fit, a normalization $S F_{\mathrm{e} \rightarrow \gamma}$ is measured and applied to both the $\mathrm{Z}+$ jets and $\mathrm{Z}+\gamma \mathrm{MC}$ events in the electron-signal regions. A corresponding $S F_{\mu \rightarrow \gamma}$ scale factor is not applied in the muon-signal regions, as the misidentification of muons as photons is negligible. The results of the fits for each of these scale factors are listed in table 2 . Comparisons of the data and $\mathrm{MC}$ distributions are shown in figure 2 after the applying the scale factors of table 2 .

The final ingredient needed to estimate the background is the relative compositions of photons and photon-like jets in the dominant $t \bar{t}+$ jets and $t \bar{t}+\gamma$ backgrounds. As stated in the introdcution, no explicit $\mathrm{t} \overline{\mathrm{t}}+\gamma \gamma$ sample is used in the background estimate because of the exceedingly small cross section for such events. The sources of two photon events in SR2 are largely the result of jets or electrons misidentified as photons as described above, or of initial or final-state radiation as predicted by PYTHIA. While the precise photon purity in each signal region is important for absolute measurements, no difference in the overall shape of simulated $p_{\mathrm{T}}^{\text {miss }}$ is found when altering the purity of selected photons. The maximum bin-by-bin difference between the simulated $p_{\mathrm{T}}^{\text {miss }}$ of $\mathrm{t} \overline{\mathrm{t}}+$ jets and $\mathrm{t} \overline{\mathrm{t}}+\gamma$ events is found to be $5 \%$. When their relative normalizations are adjusted to the observed photon purity in data through a fit to the photon isolation variable, the result is well contained within the statistical uncertainties in the $p_{\mathrm{T}}^{\text {miss }}$ distribution. The $p_{\mathrm{T}}^{\text {miss }}$ distribution in both signal regions is found to be insensitive to the source of selected photons in t $\bar{t}+$ jets and $t \bar{t}+\gamma$ backgrounds, and, as such, no dedicated $t \bar{t}+\gamma \gamma$ sample is required. To eliminate any dependence on the overall production rate of $\mathrm{t} \overline{\mathrm{t}}+\gamma$ events, the normalizations of $\mathrm{t} \overline{\mathrm{t}}+$ jets and $\mathrm{t} \overline{\mathrm{t}}+\gamma$ backgrounds are allowed to float freely in the calculations of upper limits, so that the interpretation of the results is based completely on the observed shapes of the distributions.

The control regions allow us to validate the prediction of the $p_{\mathrm{T}}^{\text {miss }}$ background, as they contain less than $1 \%$ contamination from signal. Inverting the requirements on the photon shower selection or on charged-hadron isolation, the CR1 and CR2 regions can contain the same t $\bar{t}$ systems as the signal regions, but with greatly enhanced contributions from misidentified jets compared to the photon content in each sample. The observed data and predicted background $p_{\mathrm{T}}^{\text {miss }}$ are shown in figure 3 for each control region. 

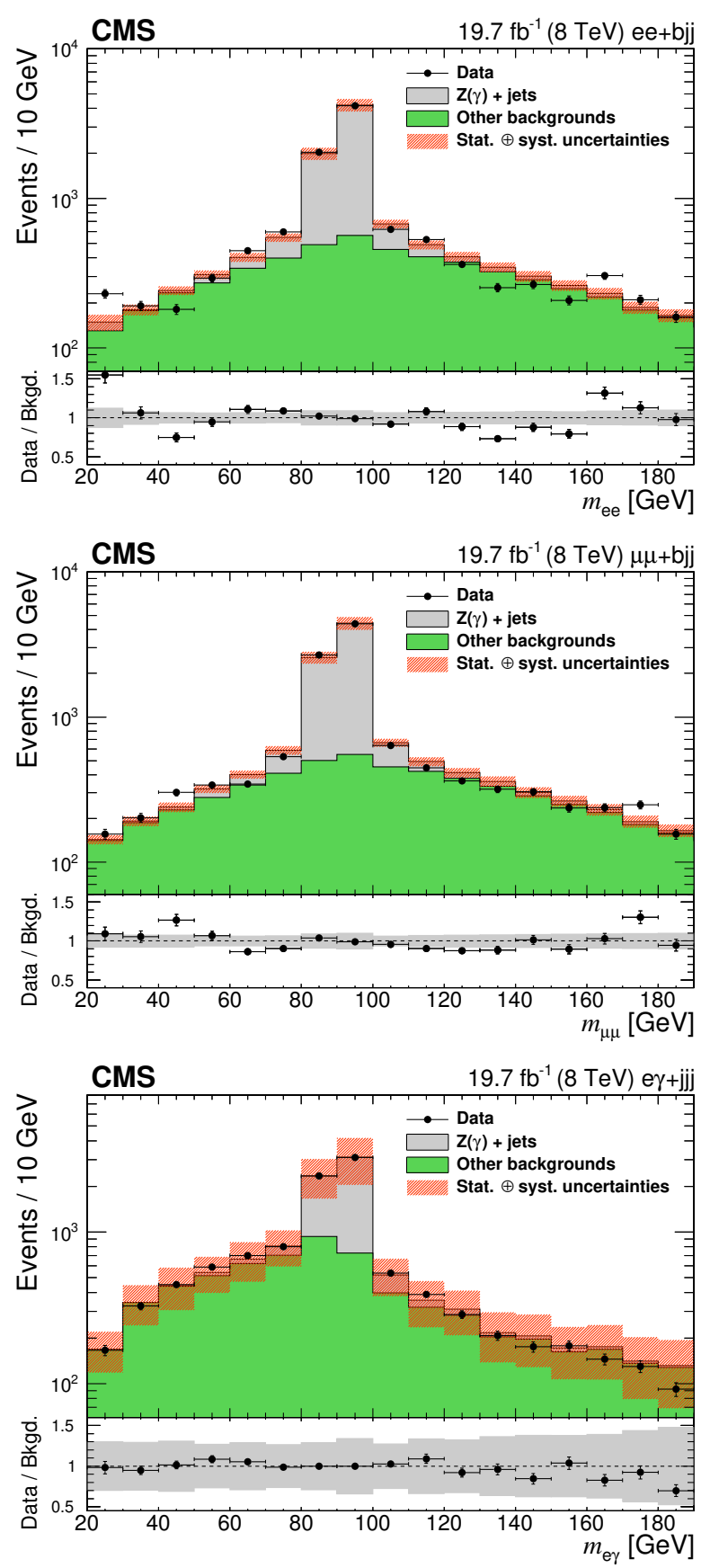

Figure 2. The dilepton invariant masses used in determining $S F_{\mathrm{Z}(\gamma)}$ (upper pane) for the electron and (middle pane) for the muon channels. The lower pane shows the result of the fit of $m_{\mathrm{e} \gamma}$ SR1 electron data (without the $\mathrm{b}$ tag requirement) to determine $S F_{\mathrm{e} \rightarrow \gamma}$. The mass spectra are shown post-fit after the application of the derived scale factors. The ratio of data to the total background is included in the lower panel of each plot. Uncertainties include the quadratic sum of all statistical and systematic components. 

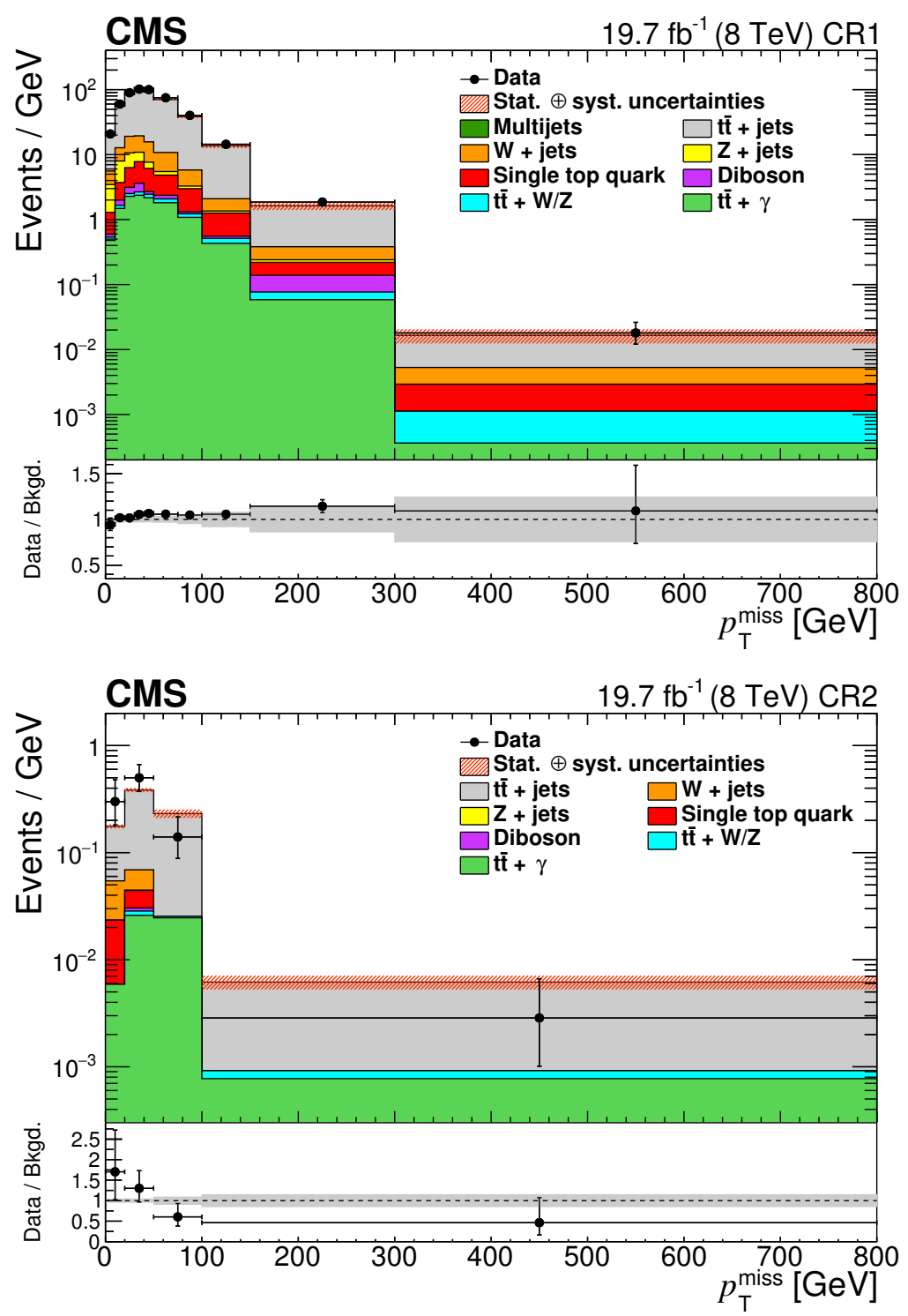

Figure 3. Comparison of data and simulated events as a function of $p_{\mathrm{T}}^{\text {miss }}$ for the combined e and $\mu$ control regions is shown: (upper pane) CR1 with one fake photon, and (lower pane) CR2 with two fake photons. The content of each bin is normalized to its bin width. The ratios of data to background are shown below the two panels. The overall uncertainties are obtained from the sum in quadrature of the statistical and systematic components. Note the Diboson background includes WW, WZ, ZZ, W+gamma, and Z+gamma. 
The bin-by-bin fractional disagreement (1-Data/Background) ranging between 10-20\% between data and background in CR1 is taken as signal region systematic uncertainty in the modeling of $p_{\mathrm{T}}^{\mathrm{miss}}$, and is applied bin-by-bin in the signal region. The KolmogorovSmirnov test [56] result of 0.66 between data and simulation for CR2 is attributable to the very small number of events in data and, therefore, CR2 is not used to determine an uncertainty for the signal region SR2. The CR1 results are therefore used for both SR1 and SR2. An additional systematic uncertainty in SR1 is obtained using the bin-bybin fractional differences $(1-\mathrm{CR} 1 / \mathrm{SR} 1)$ of $\mathrm{CR} 1$ and SR1 $p_{\mathrm{T}}^{\text {miss }}$ shapes. A final systematic uncertainty is obtained from a similar bin-by-bin difference (1-SR1/SR2) for SR2. Overall, this accounts for a 10-20\% systematic uncertainty from differences between the data and the CR1 MC $p_{\mathrm{T}}^{\text {miss }}$ shapes, a 1-8\% systematic uncertainty in SR1 due to the difference between CR1 and SR $1 p_{\mathrm{T}}^{\text {miss }}$ shapes, and a 10-50\% systematic uncertainty (the $50 \%$ value applies only in the highest bin of $p_{\mathrm{T}}^{\text {miss }}$ ) in SR2, based on the difference SR1 and SR2 $p_{\mathrm{T}}^{\mathrm{miss}}$ shapes.

\section{$5 \quad$ Results and interpretation}

For any given background or signal process, contributions from systematic uncertainties affecting $p_{\mathrm{T}}^{\text {miss }}$ are treated simultaneously and are assumed to be completely uncorrelated. All backgrounds are simulated using MC generated events and assigned systematic uncertainties based on integrated luminosity uncertainties, PDF and scale uncertainties, corrections for the number of pileup events, and jet energy scale and resolution (JES and JER). Estimated uncertainties on trigger efficiency and object selections are derived from the systematic uncertainties in MC scale factors. These include trigger efficiencies, b tagging [46, 47] as well as electron [44], muon [45], and photon identification [38]. The systematic uncertainties are summarized in table 3 .

The observed data are compared to the SM background estimates as a function of $p_{\mathrm{T}}^{\text {miss }}$ in each signal region, as shown in figure 4. No significant deviation is observed between data and the background prediction. The final results are summarized in table 4 .

To demonstated what a GGM signal would look like compared to the data, an example of a GGM spectrum is generated with FAStSim [57] using PYTHIa 6 and SUSPECT 2.41 [58], using the decay tables from SDECAY 1.2 [59] and NLO cross sections calculated with PROSPINO 2.1 [60]. We scan over the parameters $M_{1}\left(\mathrm{U}(1)_{Y}\right)$, gaugino (bino) mass and $M_{t R}$ in the SLHA files [61]. The other input parameters of GGM such as $M_{2}\left(\mathrm{SU}(2)_{L}\right)$, gaugino (wino) mass) and $M_{d R}$, etc. are decoupled. As a result SDECAY + SuSpECT produce neutralino and top squark masses that are similar to the settings of $M_{1}$ and $M_{t R}$, and the rest of the particles masses are in the $\mathrm{TeV}$ range.

The GGM signal is shown superimposed on the data and background MC in figure 4. The mass of the top squark $\left(m_{\widetilde{\mathrm{t}}}\right)$ is chosen to range from 360 to $910 \mathrm{GeV}$. The neutralino is assumed for simplicity to be $100 \%$ bino-like, decaying $100 \%$ to a photon plus a gravitino. The neutralino mass $\left(m_{\widetilde{\chi}_{1}^{0}}\right)$ is chosen to range from 150 to $725 \mathrm{GeV}$ and the gravitino mass is $1 \mathrm{GeV}$. Signal points are evaluated in $25 \mathrm{GeV}$ steps in both $m_{\widetilde{\chi}_{1}^{0}}$ and $m_{\widetilde{\mathrm{t}}}$ up to $300 \mathrm{GeV}$, and in $50 \mathrm{GeV}$ steps for higher masses. All other SUSY particles (squarks, gluinos, and 

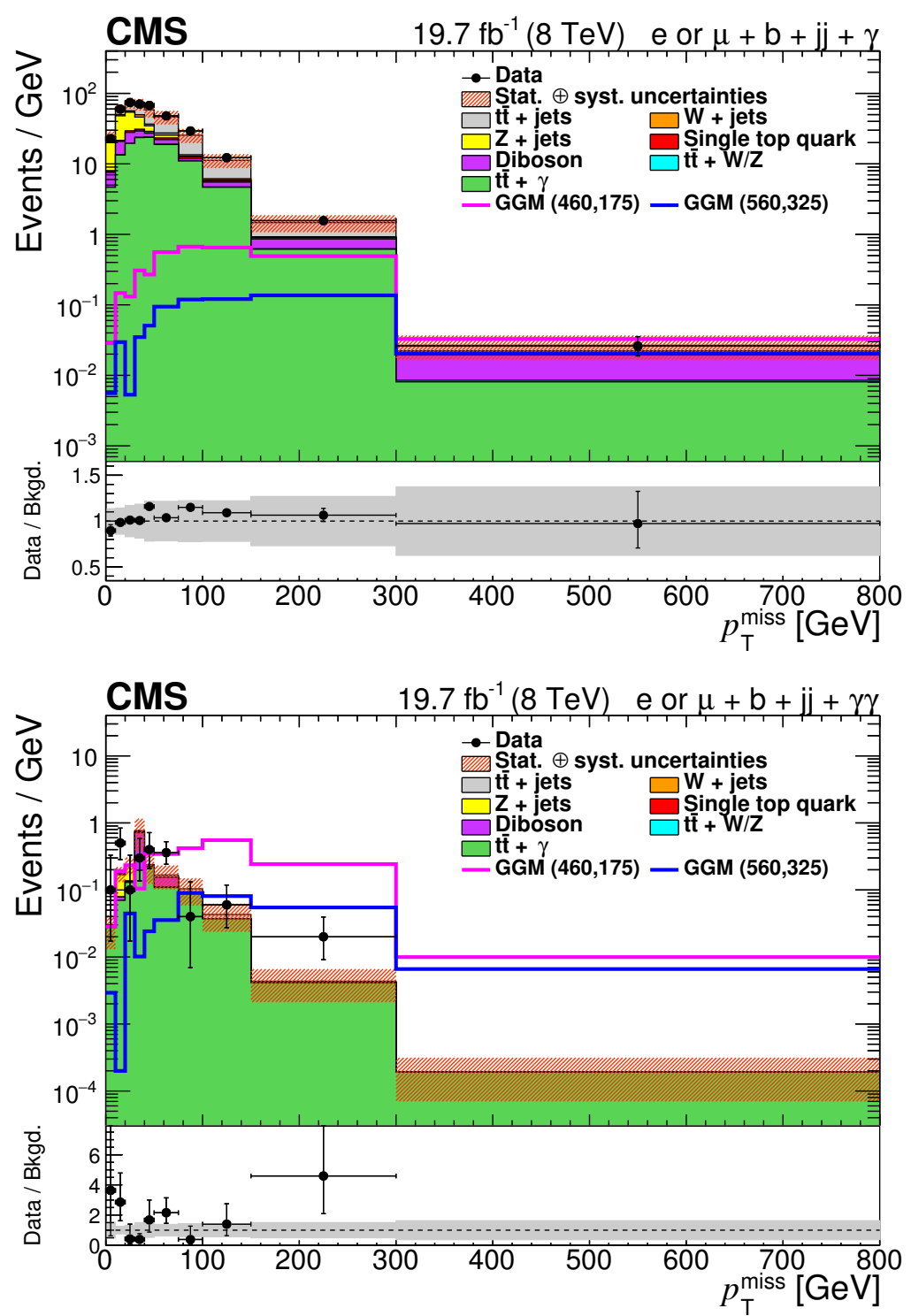

Figure 4. Comparison of data and MC simulation in $p_{\mathrm{T}}^{\text {miss }}$ for the combined (e and $\mu$ ) signal regions: (upper pane) SR1 with one reconstructed photon and (lower pane) SR2 with two reconstructed photons. Each bin is normalized by its bin width.

gauginos) are decoupled by setting their masses to very large values so that the only relevant process is the production of top squark pairs that decay to bino-like NLSPs. The mass region where $m_{\tilde{\mathrm{t}}}-m_{\widetilde{\chi}_{1}^{0}}<m_{\mathrm{t}}$ is not considered, as the requirement for high- $p_{\mathrm{T}}$ leptons and $\mathrm{b}$ jets limits the sensitivity in this mass range.

No significant excess of events is observed beyond the SM expectation, and $95 \%$ confidence level (CL) upper limits are placed on the cross sections by combining the results of all four search regions (electron SR1, muon SR1, electron SR2, and muon SR2) using the $\mathrm{CL}_{\mathrm{s}}$ criterion [62-64]. The test statistic is constructed as the product of likelihood ratios in bins of $p_{\mathrm{T}}^{\text {miss }}$. Systematic uncertainties are included as nuisance parameters in the signal 


\begin{tabular}{|lccc|}
\hline Source & Shapes & Uncertainty (\%) & Processes affected \\
\hline Integrated luminosity & & 2.6 & Signal and backgrounds \\
Lepton ID/trigger & $\checkmark$ & 1 & Signal and backgrounds \\
Photon ID & $\checkmark$ & 1.5 & Signal and backgrounds \\
Pileup & $\checkmark$ & 2 & Signal and backgrounds \\
JES/JER & $\checkmark$ & 5 & Signal and backgrounds \\
b tagging & $\checkmark$ & 2.5 & Signal and backgrounds \\
\hline Renormalization and factorization scales & & $0.5-25$ & Backgrounds \\
PDFs & & $2.5-10$ & Backgrounds \\
\hline Control region discrepancy & $\checkmark$ & $10-20$ & Backgrounds \\
SR1/CR1 shape differences & $\checkmark$ & $1-8$ & Backgrounds \\
SR2/SR1 shape differences & $\checkmark$ & $10-50$ & Backgrounds \\
\hline SUSY cross sections & & $16-28$ & Signal \\
\hline
\end{tabular}

Table 3. Summary of systematic uncertainties: the dominant uncertainties are extracted from the control region. In the calculation of the upper limits, the normalizations of the $t \bar{t}+$ jets and $\mathrm{t} \overline{\mathrm{t}}+\gamma$ backgrounds are allowed to float freely in the fit. Check marks indicate the uncertainties that affect the shape of $p_{\mathrm{T}}^{\text {miss }}$.

\begin{tabular}{|lcc|}
\hline Channel & SR1 & SR2 \\
\hline $\mathrm{t} \overline{\mathrm{t}}+$ jets & $1845 \pm 48 \pm 64$ & $1.42 \pm 1.31 \pm 0.12$ \\
$\mathrm{~W}$ or $\mathrm{Z}+$ jets & $1100 \pm 43 \pm 35$ & $2.12 \pm 1.56 \pm 0.22$ \\
Single t & $130 \pm 14 \pm 6$ & - \\
Diboson & $22.7 \pm 4.9 \pm 1.1$ & $0.20 \pm 0.44 \pm 0.06$ \\
$\mathrm{~V} \gamma$ & $431 \pm 25 \pm 116$ & $6.2 \pm 2.6 \pm 3.8$ \\
$\mathrm{t} \overline{\mathrm{t}}+\mathrm{W}$ or $\mathrm{Z}$ & $14.7 \pm 4.0 \pm 1.0$ & $0.15 \pm 0.41$ \\
$\mathrm{t} \overline{\mathrm{t}}+\gamma$ & $1926 \pm 47 \pm 388$ & $14.0 \pm 4.0 \pm 2.9$ \\
\hline Total background & $5469 \pm 85 \pm 411$ & $24.1 \pm 5.2 \pm 4.8$ \\
\hline GGM $(460,175)$ & $162 \pm 16 \pm 6$ & $87 \pm 12 \pm 2$ \\
GGM $(560,325)$ & $43.2 \pm 7.5 \pm 1.9$ & $18.1 \pm 4.8 \pm 0.6$ \\
\hline Data & 5741 & 30 \\
\hline
\end{tabular}

Table 4. Observed data and expected background yields for the combined (e and $\mu$ ) signal regions. Expectations from two GGM signal model points are included, for which $(460,175)$ refers to $m_{\mathfrak{t}}=$ $460 \mathrm{GeV}$ and $m_{\tilde{\chi}_{1}^{0}}=175 \mathrm{GeV}$, and similarly for $(560,325)$. The first group of uncertainties is statistical and the second is systematic.

and background $p_{\mathrm{T}}^{\text {miss }}$ shapes. Systematic uncertainties affecting only the normalization of signal or background processes are modeled through log-normal distributions, taken as the probability density functions in their associated nuisance parameters. Fluctuations in the shape of $p_{\mathrm{T}}^{\text {miss }}$ distributions determine both upward and downward systematic uncertainties.

A single $\pm 100 \%$ nuisance parameter is introduced with a log-uniform probability density function for its normalization to allow the $t \bar{t}$ and $t \bar{t}+\gamma$ normalizations to float freely in the upper-limit calculation. Statistical uncertainties resulting from the limited number of MC events are also included as nuisance parameters, as prescribed in ref. [65]. 

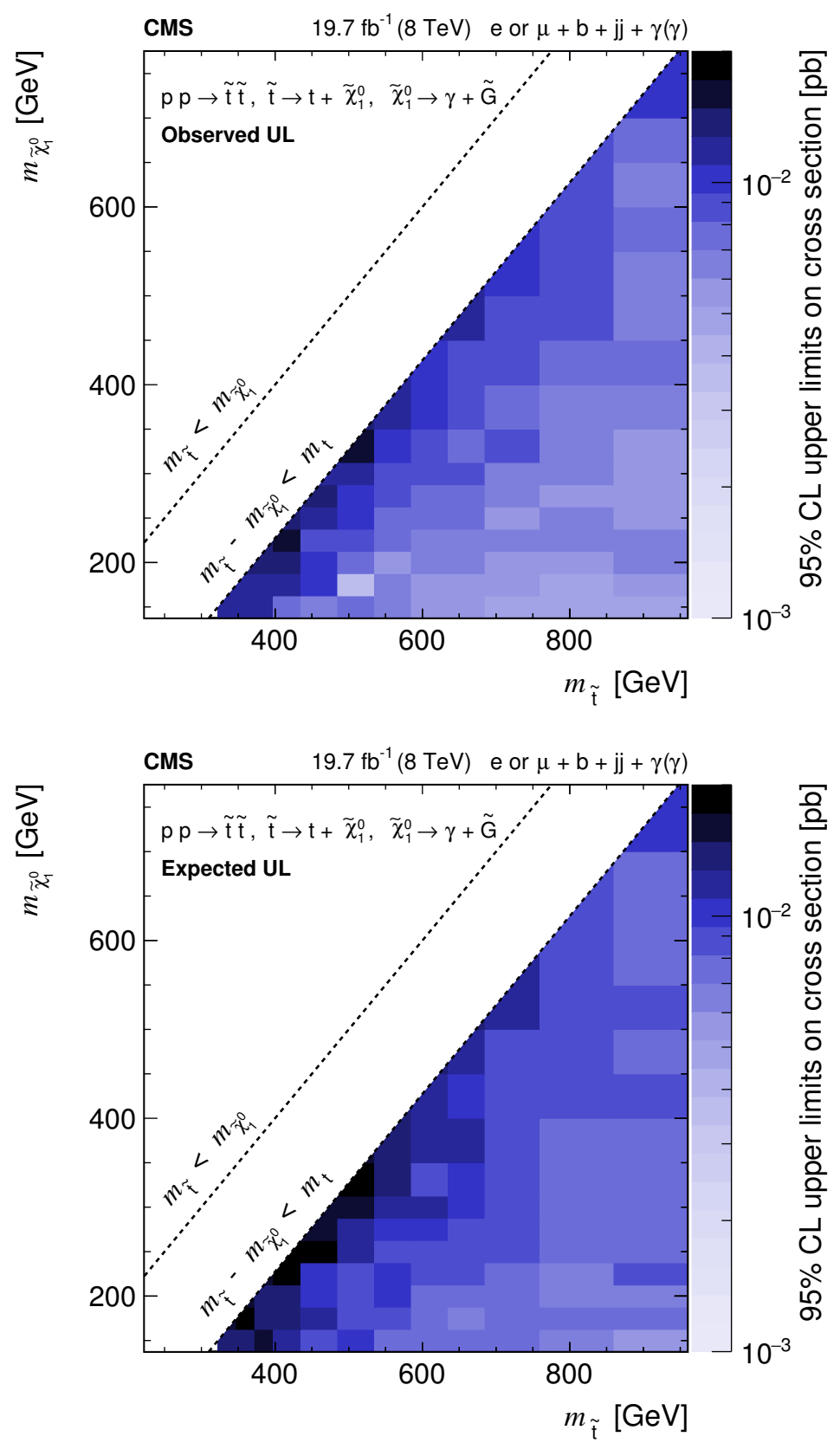

Figure 5. The observed (upper pane) and expected (lower pane) $\mathrm{CL}_{\mathrm{s}}$ upper limits on the cross section at $95 \%$ CL in the $m_{\widetilde{\mathfrak{t}}}-m_{\widetilde{\chi}_{1}^{0}}$ plane.

The expected and observed upper limits are shown in figure 5. The observed upper limits are slightly less stringent than the expected limits. Observed and expected exclusion contours are also determined and shown in figure 6 with exclusion of top squark mass below 650 to $730 \mathrm{GeV}$ corresponding to neutralino masses of 500 and $150 \mathrm{GeV}$, respectively. These exclusions are obtained using the $-1 \sigma$ theoretical excursion from the observed exclusion mean. 


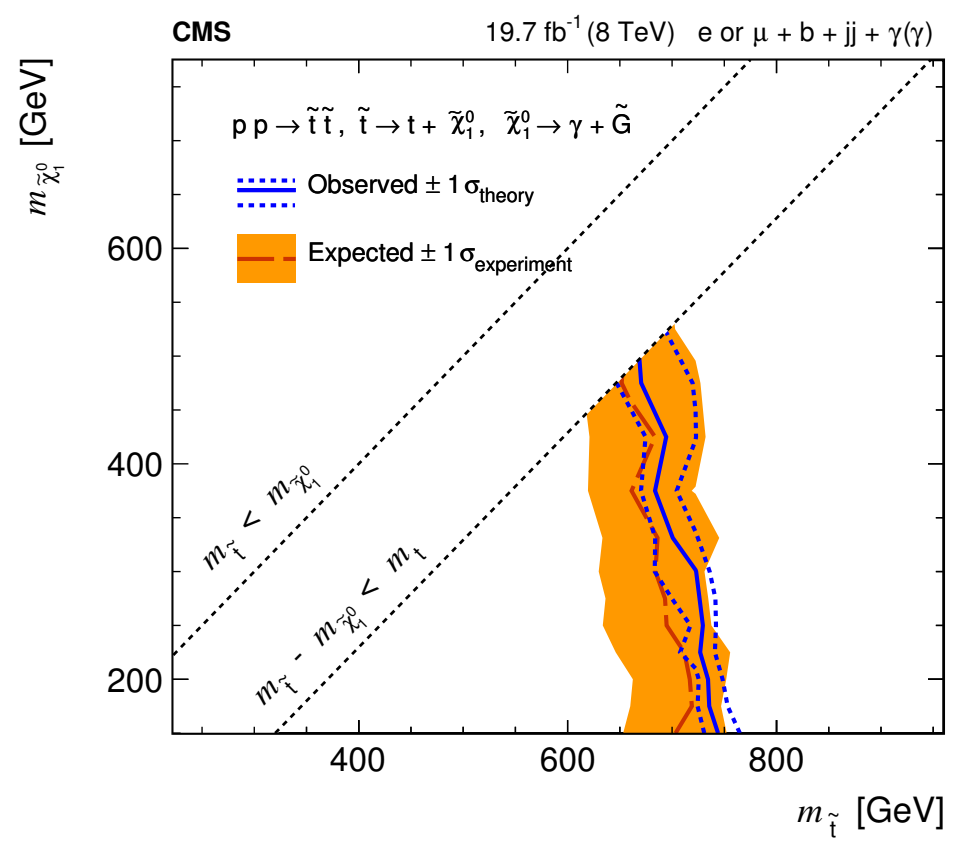

Figure 6. Observed and expected mean exclusions at the $95 \%$ CL in the top squark and bino mass plane, and their ranges of uncertainties given by the contours at the $68 \% \mathrm{CL}$. The region to the left of the contour for $\mathrm{m}_{\tilde{\mathfrak{t}}^{-}}-\mathrm{m}_{\widetilde{\chi}_{1}^{0}}<\mathrm{m}_{t}$ is excluded by this analysis.

\section{Summary}

We have presented a search for natural gauge-mediated supersymmetry breaking in events with a top quark pair and one or two photons. No significant deviation is found in the distribution of the missing transverse momentum between data and expected SM backgrounds that would indicate the presence of new physics. Upper limits on signal cross sections are calculated for a range of top squark and bino masses. Top squark masses between 650 to $730 \mathrm{GeV}$ are excluded at the $95 \%$ CL corresponding to the neutralino mass range of 500 to $150 \mathrm{GeV}$, respectively. These top squark mass points are obtained using the $-1 \sigma$ theoretical excursion from the observed exclusion mean. These results set the most stringent exclusions on top squark masses in gauge-mediated supersymmetric model considered here.

\section{Acknowledgments}

We congratulate our colleagues in the CERN accelerator departments for the excellent performance of the LHC and thank the technical and administrative staffs at CERN and at other CMS institutes for their contributions to the success of the CMS effort. In addition, we gratefully acknowledge the computing centers and personnel of the Worldwide LHC Computing Grid for delivering so effectively the computing infrastructure essential to our analyses. Finally, we acknowledge the enduring support for the construction and operation of the LHC and the CMS detector provided by the following funding agencies: 
BMWFW and FWF (Austria); FNRS and FWO (Belgium); CNPq, CAPES, FAPERJ, and FAPESP (Brazil); MES (Bulgaria); CERN; CAS, MoST, and NSFC (China); COLCIENCIAS (Colombia); MSES and CSF (Croatia); RPF (Cyprus); SENESCYT (Ecuador); MoER, ERC IUT, and ERDF (Estonia); Academy of Finland, MEC, and HIP (Finland); CEA and CNRS/IN2P3 (France); BMBF, DFG, and HGF (Germany); GSRT (Greece); OTKA and NIH (Hungary); DAE and DST (India); IPM (Iran); SFI (Ireland); INFN (Italy); MSIP and NRF (Republic of Korea); LAS (Lithuania); MOE and UM (Malaysia); BUAP, CINVESTAV, CONACYT, LNS, SEP, and UASLP-FAI (Mexico); MBIE (New Zealand); PAEC (Pakistan); MSHE and NSC (Poland); FCT (Portugal); JINR (Dubna); MON, RosAtom, RAS, RFBR and RAEP (Russia); MESTD (Serbia); SEIDI, CPAN, PCTI and FEDER (Spain); Swiss Funding Agencies (Switzerland); MST (Taipei); ThEPCenter, IPST, STAR, and NSTDA (Thailand); TUBITAK and TAEK (Turkey); NASU and SFFR (Ukraine); STFC (United Kingdom); DOE and NSF (U.S.A.).

Individuals have received support from the Marie-Curie program and the European Research Council and Horizon 2020 Grant, contract No. 675440 (European Union); the Leventis Foundation; the A. P. Sloan Foundation; the Alexander von Humboldt Foundation; the Belgian Federal Science Policy Office; the Fonds pour la Formation à la Recherche dans l'Industrie et dans l'Agriculture (FRIA-Belgium); the Agentschap voor Innovatie door Wetenschap en Technologie (IWT-Belgium); the Ministry of Education, Youth and Sports (MEYS) of the Czech Republic; the Council of Science and Industrial Research, India; the HOMING PLUS program of the Foundation for Polish Science, cofinanced from European Union, Regional Development Fund, the Mobility Plus program of the Ministry of Science and Higher Education, the National Science Center (Poland), contracts Harmonia 2014/14/M/ST2/00428, Opus 2014/13/B/ST2/02543, 2014/15/B/ST2/03998, and 2015/19/B/ST2/02861, Sonata-bis 2012/07/E/ST2/01406; the National Priorities Research Program by Qatar National Research Fund; the Programa Clarín-COFUND del Principado de Asturias; the Thalis and Aristeia programs cofinanced by EU-ESF and the Greek NSRF; the Rachadapisek Sompot Fund for Postdoctoral Fellowship, Chulalongkorn University and the Chulalongkorn Academic into Its 2nd Century Project Advancement Project (Thailand); and the Welch Foundation, contract C-1845.

Open Access. This article is distributed under the terms of the Creative Commons Attribution License (CC-BY 4.0), which permits any use, distribution and reproduction in any medium, provided the original author(s) and source are credited.

\section{References}

[1] J. Wess and B. Zumino, Supergauge transformations in four-dimensions, Nucl. Phys. B 70 (1974) 39 [INSPIRE].

[2] Yu. A. Golfand and E.P. Likhtman, Extension of the algebra of Poincaré group generators and violation of $p$ invariance, JETP Lett. 13 (1971) 323 [INSPIRE].

[3] D.V. Volkov and V.P. Akulov, Possible universal neutrino interaction, JETP Lett. 16 (1972) 438 [INSPIRE]. 
[4] A.H. Chamseddine, R.L. Arnowitt and P. Nath, Locally supersymmetric grand unification, Phys. Rev. Lett. 49 (1982) 970 [INSPIRE].

[5] G.L. Kane, C.F. Kolda, L. Roszkowski and J.D. Wells, Study of constrained minimal supersymmetry, Phys. Rev. D 49 (1994) 6173 [hep-ph/9312272] [INSPIRE].

[6] P. Fayet, Supergauge invariant extension of the Higgs mechanism and a model for the electron and its neutrino, Nucl. Phys. B 90 (1975) 104 [INSPIRE].

[7] R. Barbieri, S. Ferrara and C.A. Savoy, Gauge models with spontaneously broken local supersymmetry, Phys. Lett. 119B (1982) 343 [INSPIRE].

[8] L.J. Hall, J.D. Lykken and S. Weinberg, Supergravity as the messenger of supersymmetry breaking, Phys. Rev. D 27 (1983) 2359 [INSPIRE].

[9] P. Ramond, Dual theory for free fermions, Phys. Rev. D 3 (1971) 2415 [InSPIRE].

[10] G. 't Hooft, Naturalness, chiral symmetry, and spontaneous chiral symmetry breaking, NATO Sci. Ser. B 59 (1980) 135.

[11] E. Witten, Dynamical breaking of supersymmetry, Nucl. Phys. B 188 (1981) 513.

[12] M. Dine, W. Fischler and M. Srednicki, Supersymmetric technicolor, Nucl. Phys. B 189 (1981) 575 [INSPIRE].

[13] S. Dimopoulos and S. Raby, Supercolor, Nucl. Phys. B 192 (1981) 353 [inSPIRE].

[14] S. Dimopoulos and H. Georgi, Softly broken supersymmetry and SU(5), Nucl. Phys. B 193 (1981) 150 [INSPIRE].

[15] R.K. Kaul and P. Majumdar, Cancellation of quadratically divergent mass corrections in globally supersymmetric spontaneously broken gauge theories, Nucl. Phys. B 199 (1982) 36 [INSPIRE].

[16] ATLAS collaboration, Observation of a new particle in the search for the standard model Higgs boson with the ATLAS detector at the LHC, Phys. Lett. B 716 (2012) 1 [arXiv: 1207.7214] [INSPIRE].

[17] CMS collaboration, Observation of a new boson at a mass of $125 \mathrm{GeV}$ with the CMS experiment at the LHC, Phys. Lett. B 716 (2012) 30 [arXiv:1207.7235].

[18] ATLAS and CMS collaboration, Combined measurement of the Higgs boson mass in pp collisions at $\sqrt{s}=7$ and $8 \mathrm{TeV}$ with the ATLAS and CMS experiments, Phys. Rev. Lett. 114 (2015) 191803 [arXiv: 1503.07589] [INSPIRE].

[19] J. Barnard, B. Farmer, T. Gherghetta and M. White, Natural gauge mediation with a bino NLSP at the LHC, Phys. Rev. Lett. 109 (2012) 241801 [arXiv:1208.6062] [InSPIRE].

[20] Y. Kats, P. Meade, M. Reece and D. Shih, The status of GMSB after 1/fb at the LHC, JHEP 02 (2012) 115 [arXiv:1110.6444] [INSPIRE].

[21] CMS collaboration, Searches for pair production of third-generation squarks in $\sqrt{s}=13 \mathrm{TeV}$ pp collisions, Eur. Phys. J. C 77 (2017) 327 [arXiv: 1612.03877] [INSPIRE].

[22] CMS collaboration, Search for top squark and higgsino production using diphoton Higgs boson decays, Phys. Rev. Lett. 112 (2014) 161802 [arXiv:1312.3310] [INSPIRE].

[23] CMS collaboration, Search for top-squark pairs decaying into Higgs or $Z$ bosons in pp collisions at $\sqrt{s}=8 \mathrm{TeV}$, Phys. Lett. B 736 (2014) 371 [arXiv:1405.3886] [INSPIRE]. 
[24] ATLAS collaboration, Search for supersymmetry in events with photons, bottom quarks and missing transverse momentum in proton-proton collisions at a centre-of-mass energy of 7 TeV with the ATLAS detector, Phys. Lett. B 719 (2013) 261 [arXiv:1211.1167] [InSPIRE].

[25] ATLAS collaboration, Search for photonic signatures of gauge-mediated supersymmetry in $8 \mathrm{TeV}$ pp collisions with the ATLAS detector, Phys. Rev. D 92 (2015) 072001 [arXiv: 1507.05493] [INSPIRE].

[26] ATLAS collaboration, Search for top squarks in final states with one isolated lepton, jets and missing transverse momentum in $\sqrt{s}=13 \mathrm{TeV}$ pp collisions with the ATLAS detector, Phys. Rev. D 94 (2016) 052009 [arXiv: 1606.03903] [INSPIRE].

[27] M. Dine and W. Fischler, A phenomenological model of particle physics based on supersymmetry, Phys. Lett. 110 (1982) 227.

[28] C.R. Nappi and B.A. Ovrut, Supersymmetric extension of the $\mathrm{SU}(3) \times \mathrm{SU}(2) \times \mathrm{U}(1)$ model, Phys. Lett. 113B (1982) 175 [INSPIRE].

[29] L. Álvarez-Gaumé, M. Claudson and M.B. Wise, Low-energy supersymmetry, Nucl. Phys. B 207 (1982) 96 [INSPIRE].

[30] R. Barbier et al., R-parity violating supersymmetry, Phys. Rept. 420 (2005) 1 [hep-ph/0406039] [INSPIRE].

[31] G.R. Farrar and P. Fayet, Phenomenology of the production, decay and detection of new hadronic states associated with supersymmetry, Phys. Lett. 76B (1978) 575 [INSPIRE].

[32] P. Meade, N. Seiberg and D. Shih, General gauge mediation, Prog. Theor. Phys. Suppl. 177 (2009) 143 [arXiv:0801.3278] [INSPIRE].

[33] M. Buican, P. Meade, N. Seiberg and D. Shih, Exploring general gauge mediation, JHEP 03 (2009) 016 [arXiv:0812.3668] [InSPIRE].

[34] S. Abel, M.J. Dolan, J. Jaeckel and V.V. Khoze, Phenomenology of pure general gauge mediation, JHEP 12 (2009) 001 [arXiv:0910.2674] [INSPIRE].

[35] J.T. Ruderman and D. Shih, General neutralino NLSPs at the early LHC, JHEP 08 (2012) 159 [arXiv: 1103.6083] [INSPIRE].

[36] Y. Kats and M.J. Strassler, Probing colored particles with photons, leptons and jets, JHEP 11 (2012) 097 [Erratum ibid. 07 (2016) 009] [arXiv: 1204.1119] [InSPIRE].

[37] P. Grajek, A. Mariotti and D. Redigolo, Phenomenology of general gauge mediation in light of a 125 GeV Higgs, JHEP 07 (2013) 109 [arXiv: 1303.0870] [INSPIRE].

[38] CMS collaboration, Performance of photon reconstruction and identification with the CMS detector in proton-proton collisions at $\sqrt{s}=8 \mathrm{TeV}, 2015$ JINST $10 \mathrm{P} 08010$ [arXiv: 1502.02702] [INSPIRE].

[39] CMS collaboration, The CMS experiment at the CERN LHC, 2008 JINST 3 S08004 [INSPIRE].

[40] CMS collaboration, Particle-flow reconstruction and global event description with the CMS detector, 2017 JINST 12 P10003 [arXiv:1706.04965] [INSPIRE].

[41] CMS collaboration, Commissioning of the particle-flow reconstruction in minimum-bias and jet events from pp collisions at $7 \mathrm{TeV}$, CMS-PAS-PFT-10-002 (2010).

[42] M. Cacciari, G.P. Salam and G. Soyez, The anti- $k_{t}$ jet clustering algorithm, JHEP 04 (2008) 063 [arXiv: 0802.1189] [INSPIRE]. 
[43] M. Cacciari, G.P. Salam and G. Soyez, FastJet user manual, Eur. Phys. J. C 72 (2012) 1896 [arXiv:1111.6097] [INSPIRE].

[44] CMS collaboration, Performance of electron reconstruction and selection with the CMS detector in proton-proton collisons at $\sqrt{(s)}=8 \mathrm{TeV}, 2006$ JINST $10 \mathrm{P} 06005$.

[45] CMS collaboration, Performance of CMS muon reconstruction in pp collision events at $\sqrt{s}=7 \mathrm{TeV}, 2012$ JINST 7 P10002 [arXiv:1206.4071] [INSPIRE].

[46] CMS collaboration, Identification of b-quarks jets with the CMS experiment, CMS-PAS-BTV-12-001 (2012).

[47] CMS collaboration, Performance of $b$ tagging at $\sqrt{s}=8 \mathrm{TeV}$ in multijet, $t \bar{t}$ and boosted topology events, CMS-PAS-BTV-13-001 (2013).

[48] J. Alwall, M. Herquet, F. Maltoni, O. Mattelaer and T. Stelzer, MadGraph 5: going beyond, JHEP 06 (2011) 128 [arXiv:1106.0522] [INSPIRE].

[49] T. Sjöstrand, S. Mrenna and P.Z. Skands, PYTHIA 6.4 physics and manual, JHEP 05 (2006) 026 [hep-ph/0603175] [inSPIRE].

[50] S. Frixione, P. Nason and C. Oleari, Matching NLO QCD computations with Parton Shower simulations: the POWHEG method, JHEP 11 (2007) 070 [arXiv:0709.2092] [INSPIRE].

[51] Z. Was, TAUOLA the library for tau lepton decay and KKMC/KORALB/KORALZ/... status report, Nucl. Phys. Proc. Suppl. 98 (2001) 96 [hep-ph/0011305] [InSPIRE].

[52] CMS collaboration, Study of the underlying event at forward rapidity in pp collisions at $\sqrt{s}=0.9,2.76$ and $7 \mathrm{TeV}$, JHEP 04 (2013) 072 [arXiv: 1302.2394] [inSPIRE].

[53] CMS collaboration, Event generator tunes obtained from underlying event and multiparton scattering measurements, Eur. Phys. J. C 76 (2016) 155 [arXiv:1512.00815] [INSPIRE].

[54] GEANT4 collaboration, S. Agostinelli et al., GEANT4 - a simulation toolkit, Nucl. Instrum. Meth. A 506 (2003) 250 [INSPIRE].

[55] J. Pumplin et al., New generation of parton distributions with uncertainties from global QCD analysis, JHEP 07 (2002) 012 [hep-ph/0201195] [INSPIRE].

[56] N. Smirnov, Table for estimating the goodness of fit of empirical distributions, Ann. Math. Statist. 19 (1948) 279.

[57] S. Abdullin et al., The fast simulation of the CMS detector at LHC, J. Phys. Conf. Ser. 331 (2011) 032049.

[58] A. Djouadi, J.-L. Kneur and G. Moultaka, SuSpect: a Fortran code for the supersymmetric and Higgs particle spectrum in the MSSM, Comput. Phys. Commun. 176 (2007) 426 [hep-ph/0211331] [INSPIRE].

[59] M. Muhlleitner, A. Djouadi and Y. Mambrini, SDECAY: a Fortran code for the decays of the supersymmetric particles in the MSSM, Comput. Phys. Commun. 168 (2005) 46 [hep-ph/0311167] [INSPIRE].

[60] W. Beenakker, R. Hopker and M. Spira, PROSPINO: a program for the production of supersymmetric particles in next-to-leading order QCD, hep-ph/9611232 [INSPIRE].

[61] B.C. Allanach et al., SUSY Les Houches accord 2, Comput. Phys. Commun. 180 (2009) 8 [arXiv:0801.0045] [INSPIRE]. 
[62] T. Junk, Confidence level computation for combining searches with small statistics, Nucl. Instrum. Meth. A 434 (1999) 435 [hep-ex/9902226].

[63] A.L. Read, Presentation of search results: the CL(s) technique, J. Phys. G 28 (2002) 2693 [INSPIRE].

[64] G. Cowan, K. Cranmer, E. Gross and O. Vitells, Asymptotic formulae for likelihood-based tests of new physics, Eur. Phys. J. C 71 (2011) 1554 [Erratum ibid. C 73 (2013) 2501] [arXiv: 1007.1727] [INSPIRE].

[65] R.J. Barlow and C. Beeston, Fitting using finite Monte Carlo samples, Comput. Phys. Commun. 77 (1993) 219 [INSPIRE]. 


\section{The CMS collaboration}

\section{Yerevan Physics Institute, Yerevan, Armenia}

A.M. Sirunyan, A. Tumasyan

\section{Institut für Hochenergiephysik, Wien, Austria}

W. Adam, E. Asilar, T. Bergauer, J. Brandstetter, E. Brondolin, M. Dragicevic, J. Erö, M. Flechl, M. Friedl, R. Frühwirth ${ }^{1}$, V.M. Ghete, C. Hartl, N. Hörmann, J. Hrubec, M. Jeitler ${ }^{1}$, A. König, I. Krätschmer, D. Liko, T. Matsushita, I. Mikulec, D. Rabady, N. Rad, B. Rahbaran, H. Rohringer, J. Schieck ${ }^{1}$, J. Strauss, W. Waltenberger, C.-E. Wulz ${ }^{1}$

Institute for Nuclear Problems, Minsk, Belarus

O. Dvornikov, V. Makarenko, V. Mossolov, J. Suarez Gonzalez, V. Zykunov

National Centre for Particle and High Energy Physics, Minsk, Belarus

N. Shumeiko

\section{Universiteit Antwerpen, Antwerpen, Belgium}

S. Alderweireldt, E.A. De Wolf, X. Janssen, J. Lauwers, M. Van De Klundert, H. Van Haevermaet, P. Van Mechelen, N. Van Remortel, A. Van Spilbeeck

\section{Vrije Universiteit Brussel, Brussel, Belgium}

S. Abu Zeid, F. Blekman, J. D'Hondt, N. Daci, I. De Bruyn, K. Deroover, S. Lowette, S. Moortgat, L. Moreels, A. Olbrechts, Q. Python, K. Skovpen, S. Tavernier, W. Van Doninck, P. Van Mulders, I. Van Parijs

Université Libre de Bruxelles, Bruxelles, Belgium

H. Brun, B. Clerbaux, G. De Lentdecker, H. Delannoy, G. Fasanella, L. Favart, R. Goldouzian, A. Grebenyuk, G. Karapostoli, T. Lenzi, A. Léonard, J. Luetic, T. Maerschalk, A. Marinov, A. Randle-conde, T. Seva, C. Vander Velde, P. Vanlaer, D. Vannerom, R. Yonamine, F. Zenoni, F. Zhang ${ }^{2}$

\section{Ghent University, Ghent, Belgium}

T. Cornelis, D. Dobur, A. Fagot, M. Gul, I. Khvastunov, D. Poyraz, S. Salva, R. Schöfbeck, M. Tytgat, W. Van Driessche, W. Verbeke, N. Zaganidis

\section{Université Catholique de Louvain, Louvain-la-Neuve, Belgium}

H. Bakhshiansohi, O. Bondu, S. Brochet, G. Bruno, A. Caudron, S. De Visscher, C. Delaere, M. Delcourt, B. Francois, A. Giammanco, A. Jafari, M. Komm, G. Krintiras, V. Lemaitre, A. Magitteri, A. Mertens, M. Musich, K. Piotrzkowski, L. Quertenmont, M. Vidal Marono, S. Wertz

\section{Université de Mons, Mons, Belgium}

N. Beliy

\section{Centro Brasileiro de Pesquisas Fisicas, Rio de Janeiro, Brazil}

W.L. Aldá Júnior, F.L. Alves, G.A. Alves, L. Brito, C. Hensel, A. Moraes, M.E. Pol, P. Rebello Teles 
Universidade do Estado do Rio de Janeiro, Rio de Janeiro, Brazil

E. Belchior Batista Das Chagas, W. Carvalho, J. Chinellato ${ }^{3}$, A. Custódio, E.M. Da Costa, G.G. Da Silveira ${ }^{4}$, D. De Jesus Damiao, C. De Oliveira Martins, S. Fonseca De Souza, L.M. Huertas Guativa, H. Malbouisson, D. Matos Figueiredo, C. Mora Herrera, L. Mundim, H. Nogima, W.L. Prado Da Silva, A. Santoro, A. Sznajder, E.J. Tonelli Manganote ${ }^{3}$, F. Torres Da Silva De Araujo, A. Vilela Pereira

Universidade Estadual Paulista ${ }^{a}$, Universidade Federal do ABC ${ }^{b}$, São Paulo, Brazil

S. Ahuja ${ }^{a}$, C.A. Bernardes ${ }^{a}$, S. Dogra ${ }^{a}$, T.R. Fernandez Perez Tomei ${ }^{a}$, E.M. Gregores ${ }^{b}$, P.G. Mercadante ${ }^{b}$, C.S. Moon ${ }^{a}$, S.F. Novaes ${ }^{a}$, Sandra S. Padula ${ }^{a}$, D. Romero Abad ${ }^{b}$, J.C. Ruiz Vargas ${ }^{a}$

Institute for Nuclear Research and Nuclear Energy of Bulgaria Academy of Sciences

A. Aleksandrov, R. Hadjiiska, P. Iaydjiev, M. Rodozov, S. Stoykova, G. Sultanov, M. Vutova

University of Sofia, Sofia, Bulgaria

A. Dimitrov, I. Glushkov, L. Litov, B. Pavlov, P. Petkov

Beihang University, Beijing, China

W. Fang ${ }^{5}$, X. Gao $^{5}$

Institute of High Energy Physics, Beijing, China

M. Ahmad, J.G. Bian, G.M. Chen, H.S. Chen, M. Chen, Y. Chen, T. Cheng, C.H. Jiang,

D. Leggat, Z. Liu, F. Romeo, M. Ruan, S.M. Shaheen, A. Spiezia, J. Tao, C. Wang, Z. Wang, E. Yazgan, H. Zhang, J. Zhao

State Key Laboratory of Nuclear Physics and Technology, Peking University, Beijing, China

Y. Ban, G. Chen, Q. Li, S. Liu, Y. Mao, S.J. Qian, D. Wang, Z. Xu

Universidad de Los Andes, Bogota, Colombia

C. Avila, A. Cabrera, L.F. Chaparro Sierra, C. Florez, J.P. Gomez, C.F. González Hernández, J.D. Ruiz Alvarez ${ }^{6}$, J.C. Sanabria

University of Split, Faculty of Electrical Engineering, Mechanical Engineering and Naval Architecture, Split, Croatia

N. Godinovic, D. Lelas, I. Puljak, P.M. Ribeiro Cipriano, T. Sculac

University of Split, Faculty of Science, Split, Croatia

Z. Antunovic, M. Kovac

Institute Rudjer Boskovic, Zagreb, Croatia

V. Brigljevic, D. Ferencek, K. Kadija, B. Mesic, T. Susa 
University of Cyprus, Nicosia, Cyprus

M.W. Ather, A. Attikis, G. Mavromanolakis, J. Mousa, C. Nicolaou, F. Ptochos, P.A. Razis, H. Rykaczewski

Charles University, Prague, Czech Republic

M. Finger ${ }^{7}$, M. Finger Jr. ${ }^{7}$

Universidad San Francisco de Quito, Quito, Ecuador

E. Carrera Jarrin

Academy of Scientific Research and Technology of the Arab Republic of Egypt, Egyptian Network of High Energy Physics, Cairo, Egypt

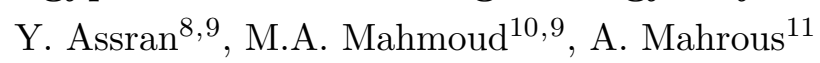

National Institute of Chemical Physics and Biophysics, Tallinn, Estonia

M. Kadastik, L. Perrini, M. Raidal, A. Tiko, C. Veelken

Department of Physics, University of Helsinki, Helsinki, Finland

P. Eerola, J. Pekkanen, M. Voutilainen

Helsinki Institute of Physics, Helsinki, Finland

J. Härkönen, T. Järvinen, V. Karimäki, R. Kinnunen, T. Lampén, K. Lassila-Perini, S. Lehti, T. Lindén, P. Luukka, J. Tuominiemi, E. Tuovinen, L. Wendland

Lappeenranta University of Technology, Lappeenranta, Finland

J. Talvitie, T. Tuuva

IRFU, CEA, Université Paris-Saclay, Gif-sur-Yvette, France

M. Besancon, F. Couderc, M. Dejardin, D. Denegri, B. Fabbro, J.L. Faure, C. Favaro, F. Ferri, S. Ganjour, S. Ghosh, A. Givernaud, P. Gras, G. Hamel de Monchenault, P. Jarry, I. Kucher, E. Locci, M. Machet, J. Malcles, J. Rander, A. Rosowsky, M. Titov

Laboratoire Leprince-Ringuet, Ecole polytechnique, CNRS/IN2P3, Université Paris-Saclay, Palaiseau, France

A. Abdulsalam, I. Antropov, S. Baffioni, F. Beaudette, P. Busson, L. Cadamuro, E. Chapon, C. Charlot, O. Davignon, R. Granier de Cassagnac, M. Jo, S. Lisniak, A. Lobanov, P. Miné, M. Nguyen, C. Ochando, G. Ortona, P. Paganini, P. Pigard, S. Regnard, R. Salerno, Y. Sirois, A.G. Stahl Leiton, T. Strebler, Y. Yilmaz, A. Zabi, A. Zghiche

Université de Strasbourg, CNRS, IPHC UMR 7178, F-67000 Strasbourg, France

J.-L. Agram ${ }^{12}$, J. Andrea, D. Bloch, J.-M. Brom, M. Buttignol, E.C. Chabert, N. Chanon, C. Collard, E. Conte ${ }^{12}$, X. Coubez, J.-C. Fontaine ${ }^{12}$, D. Gelé, U. Goerlach, A.-C. Le Bihan, P. Van Hove

Centre de Calcul de l'Institut National de Physique Nucleaire et de Physique des Particules, CNRS/IN2P3, Villeurbanne, France

S. Gadrat 
Université de Lyon, Université Claude Bernard Lyon 1, CNRS-IN2P3, Institut de Physique Nucléaire de Lyon, Villeurbanne, France

S. Beauceron, C. Bernet, G. Boudoul, C.A. Carrillo Montoya, R. Chierici, D. Contardo, B. Courbon, P. Depasse, H. El Mamouni, J. Fay, L. Finco, S. Gascon, M. Gouzevitch, G. Grenier, B. Ille, F. Lagarde, I.B. Laktineh, M. Lethuillier, L. Mirabito, A.L. Pequegnot, S. Perries, A. Popov ${ }^{13}$, V. Sordini, M. Vander Donckt, P. Verdier, S. Viret

Georgian Technical University, Tbilisi, Georgia

A. Khvedelidze ${ }^{7}$

Tbilisi State University, Tbilisi, Georgia

Z. Tsamalaidze ${ }^{7}$

RWTH Aachen University, I. Physikalisches Institut, Aachen, Germany

C. Autermann, S. Beranek, L. Feld, M.K. Kiesel, K. Klein, M. Lipinski, M. Preuten, C. Schomakers, J. Schulz, T. Verlage

RWTH Aachen University, III. Physikalisches Institut A, Aachen, Germany

A. Albert, M. Brodski, E. Dietz-Laursonn, D. Duchardt, M. Endres, M. Erdmann, S. Erdweg, T. Esch, R. Fischer, A. Güth, M. Hamer, T. Hebbeker, C. Heidemann, K. Hoepfner, S. Knutzen, M. Merschmeyer, A. Meyer, P. Millet, S. Mukherjee, M. Olschewski,

K. Padeken, T. Pook, M. Radziej, H. Reithler, M. Rieger, F. Scheuch, L. Sonnenschein,

D. Teyssier, S. Thüer

RWTH Aachen University, III. Physikalisches Institut B, Aachen, Germany

V. Cherepanov, G. Flügge, B. Kargoll, T. Kress, A. Künsken, J. Lingemann, T. Müller, A. Nehrkorn, A. Nowack, C. Pistone, O. Pooth, A. Stahl ${ }^{14}$

Deutsches Elektronen-Synchrotron, Hamburg, Germany

M. Aldaya Martin, T. Arndt, C. Asawatangtrakuldee, K. Beernaert, O. Behnke, U. Behrens, A.A. Bin Anuar, K. Borras ${ }^{15}$, A. Campbell, P. Connor, C. ContrerasCampana, F. Costanza, C. Diez Pardos, G. Dolinska, G. Eckerlin, D. Eckstein, T. Eichhorn, E. Eren, E. Gallo ${ }^{16}$, J. Garay Garcia, A. Geiser, A. Gizhko, J.M. Grados Luyando, A. Grohsjean, P. Gunnellini, A. Harb, J. Hauk, M. Hempel ${ }^{17}$, H. Jung, A. Kalogeropoulos, O. Karacheban ${ }^{17}$, M. Kasemann, J. Keaveney, C. Kleinwort, I. Korol, D. Krücker, W. Lange, A. Lelek, T. Lenz, J. Leonard, K. Lipka, W. Lohmann ${ }^{17}$, R. Mankel, I.A. Melzer-Pellmann, A.B. Meyer, G. Mittag, J. Mnich, A. Mussgiller, E. Ntomari, D. Pitzl, R. Placakyte, A. Raspereza, B. Roland, M.Ö. Sahin, P. Saxena, T. Schoerner-Sadenius, S. Spannagel, N. Stefaniuk, G.P. Van Onsem, R. Walsh, C. Wissing

\section{University of Hamburg, Hamburg, Germany}

V. Blobel, M. Centis Vignali, A.R. Draeger, T. Dreyer, E. Garutti, D. Gonzalez, J. Haller, M. Hoffmann, A. Junkes, R. Klanner, R. Kogler, N. Kovalchuk, S. Kurz, T. Lapsien, I. Marchesini, D. Marconi, M. Meyer, M. Niedziela, D. Nowatschin, F. Pantaleo ${ }^{14}$, T. Peiffer, A. Perieanu, C. Scharf, P. Schleper, A. Schmidt, S. Schumann, J. Schwandt, J. Sonneveld, H. Stadie, G. Steinbrück, F.M. Stober, M. Stöver, H. Tholen, D. Troendle, E. Usai, L. Vanelderen, A. Vanhoefer, B. Vormwald 
Institut für Experimentelle Kernphysik, Karlsruhe, Germany

M. Akbiyik, C. Barth, S. Baur, C. Baus, J. Berger, E. Butz, R. Caspart, T. Chwalek,

F. Colombo, W. De Boer, A. Dierlamm, S. Fink, B. Freund, R. Friese, M. Giffels, A. Gilbert,

P. Goldenzweig, D. Haitz, F. Hartmann ${ }^{14}$, S.M. Heindl, U. Husemann, F. Kassel ${ }^{14}$,

I. Katkov ${ }^{13}$, S. Kudella, H. Mildner, M.U. Mozer, Th. Müller, M. Plagge, G. Quast,

K. Rabbertz, S. Röcker, F. Roscher, M. Schröder, I. Shvetsov, G. Sieber, H.J. Simonis,

R. Ulrich, S. Wayand, M. Weber, T. Weiler, S. Williamson, C. Wöhrmann, R. Wolf

Institute of Nuclear and Particle Physics (INPP), NCSR Demokritos, Aghia Paraskevi, Greece

G. Anagnostou, G. Daskalakis, T. Geralis, V.A. Giakoumopoulou, A. Kyriakis, D. Loukas,

I. Topsis-Giotis

National and Kapodistrian University of Athens, Athens, Greece

S. Kesisoglou, A. Panagiotou, N. Saoulidou, E. Tziaferi

National Technical University of Athens, Athens, Greece

K. Kousouris

University of Ioánnina, Ioánnina, Greece

I. Evangelou, G. Flouris, C. Foudas, P. Kokkas, N. Loukas, N. Manthos, I. Papadopoulos,

E. Paradas, F.A. Triantis

MTA-ELTE Lendület CMS Particle and Nuclear Physics Group, Eötvös Loránd University, Budapest, Hungary

N. Filipovic, G. Pasztor

Wigner Research Centre for Physics, Budapest, Hungary

G. Bencze, C. Hajdu, D. Horvath ${ }^{18}$, F. Sikler, V. Veszpremi, G. Vesztergombi ${ }^{19}$, A.J. Zsigmond

Institute of Nuclear Research ATOMKI, Debrecen, Hungary

N. Beni, S. Czellar, J. Karancsi ${ }^{20}$, A. Makovec, J. Molnar, Z. Szillasi

Institute of Physics, University of Debrecen, Debrecen, Hungary

M. Bartók ${ }^{19}$, P. Raics, Z.L. Trocsanyi, B. Ujvari

Indian Institute of Science (IISc), Bangalore, India

S. Choudhury, J.R. Komaragiri

National Institute of Science Education and Research, Bhubaneswar, India

S. Bahinipati ${ }^{21}$, S. Bhowmik ${ }^{22}$, P. Mal, K. Mandal, A. Nayak ${ }^{23}$, D.K. Sahoo ${ }^{21}$, N. Sahoo, S.K. Swain

Panjab University, Chandigarh, India

S. Bansal, S.B. Beri, V. Bhatnagar, U. Bhawandeep, R. Chawla, A.K. Kalsi, A. Kaur, M. Kaur, R. Kumar, P. Kumari, A. Mehta, M. Mittal, J.B. Singh, G. Walia 
University of Delhi, Delhi, India

Ashok Kumar, A. Bhardwaj, B.C. Choudhary, R.B. Garg, S. Keshri, A. Kumar, S. Malhotra, M. Naimuddin, K. Ranjan, R. Sharma, V. Sharma

Saha Institute of Nuclear Physics, HBNI, Kolkata, India

R. Bhattacharya, S. Bhattacharya, K. Chatterjee, S. Dey, S. Dutt, S. Dutta, S. Ghosh, N. Majumdar, A. Modak, K. Mondal, S. Mukhopadhyay, S. Nandan, A. Purohit, A. Roy, D. Roy, S. Roy Chowdhury, S. Sarkar, M. Sharan, S. Thakur

Indian Institute of Technology Madras, Madras, India

P.K. Behera

Bhabha Atomic Research Centre, Mumbai, India

R. Chudasama, D. Dutta, V. Jha, V. Kumar, A.K. Mohanty ${ }^{14}$, P.K. Netrakanti, L.M. Pant, P. Shukla, A. Topkar

Tata Institute of Fundamental Research-A, Mumbai, India

T. Aziz, S. Dugad, G. Kole, B. Mahakud, S. Mitra, G.B. Mohanty, B. Parida, N. Sur, B. Sutar

Tata Institute of Fundamental Research-B, Mumbai, India

S. Banerjee, R.K. Dewanjee, S. Ganguly, M. Guchait, Sa. Jain, S. Kumar, M. Maity ${ }^{22}$, G. Majumder, K. Mazumdar, T. Sarkar ${ }^{22}$, N. Wickramage ${ }^{24}$

Indian Institute of Science Education and Research (IISER), Pune, India

S. Chauhan, S. Dube, V. Hegde, A. Kapoor, K. Kothekar, S. Pandey, A. Rane, S. Sharma

Institute for Research in Fundamental Sciences (IPM), Tehran, Iran

S. Chenarani ${ }^{25}$, E. Eskandari Tadavani, S.M. Etesami ${ }^{25}$, M. Khakzad, M. Mohammadi Najafabadi, M. Naseri, S. Paktinat Mehdiabadi ${ }^{26}$, F. Rezaei Hosseinabadi, B. Safarzadeh ${ }^{27}$, M. Zeinali

University College Dublin, Dublin, Ireland

M. Felcini, M. Grunewald

INFN Sezione di Bari ${ }^{a}$, Università di Bari ${ }^{b}$, Politecnico di Bari ${ }^{c}$, Bari, Italy M. Abbrescia ${ }^{a, b}$, C. Calabria ${ }^{a, b}$, C. Caputo ${ }^{a, b}$, A. Colaleo $^{a}$, D. Creanza ${ }^{a, c}$, L. Cristella $^{a, b}$, N. De Filippis ${ }^{a, c}$, M. De Palma ${ }^{a, b}$, L. Fiore ${ }^{a}$, G. Iaselli ${ }^{a, c}$, G. Maggi ${ }^{a, c}$, M. Maggi $^{a}$, G. Miniello ${ }^{a, b}$, S. My ${ }^{a, b}$, S. Nuzzo ${ }^{a, b}$, A. Pompili ${ }^{a, b}$, G. Pugliese ${ }^{a, c}$, R. Radogna ${ }^{a, b}$,

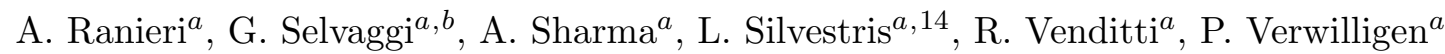

INFN Sezione di Bologna ${ }^{a}$, Università di Bologna ${ }^{b}$, Bologna, Italy

G. Abbiendi ${ }^{a}$, C. Battilana, D. Bonacorsi ${ }^{a}, b$, S. Braibant-Giacomelli ${ }^{a}, b$, L. Brigliadori ${ }^{a}, b$, R. Campanini ${ }^{a, b}$, P. Capiluppi ${ }^{a, b}$, A. Castro ${ }^{a, b}$, F.R. Cavallo ${ }^{a}$, S.S. Chhibra ${ }^{a, b}$,

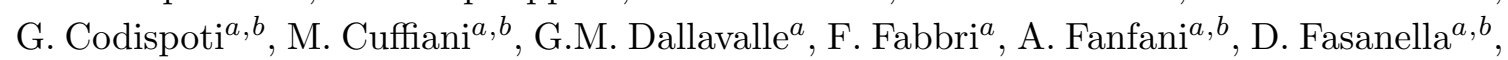
P. Giacomelli ${ }^{a}$, C. Grandi ${ }^{a}$, L. Guiducci ${ }^{a}, b$, S. Marcellini ${ }^{a}$, G. Masetti ${ }^{a}$, A. Montanari ${ }^{a}$,

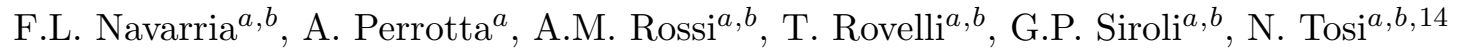


INFN Sezione di Catania ${ }^{a}$, Università di Catania ${ }^{b}$, Catania, Italy

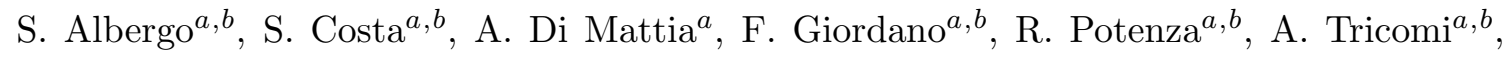

C. Tuve ${ }^{a, b}$

INFN Sezione di Firenze ${ }^{a}$, Università di Firenze ${ }^{b}$, Firenze, Italy

G. Barbagli ${ }^{a}$, V. Ciulli ${ }^{a} b$, C. Civinini ${ }^{a}$, R. D'Alessandro ${ }^{a, b}$, E. Focardi $^{a, b}$, P. Lenzi $^{a, b}$,

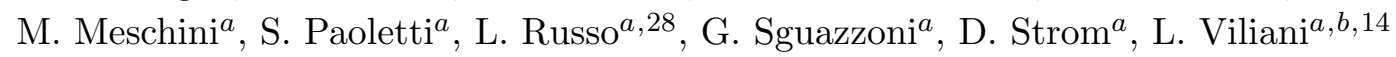

INFN Laboratori Nazionali di Frascati, Frascati, Italy

L. Benussi, S. Bianco, F. Fabbri, D. Piccolo, F. Primavera ${ }^{14}$

INFN Sezione di Genova ${ }^{a}$, Università di Genova ${ }^{b}$, Genova, Italy

V. Calvelli ${ }^{a, b}$, F. Ferro ${ }^{a}$, M.R. Monge ${ }^{a, b}$, E. Robutti ${ }^{a}$, S. Tosi ${ }^{a, b}$

INFN Sezione di Milano-Bicocca ${ }^{a}$, Università di Milano-Bicocca ${ }^{b}$, Milano, Italy

L. Brianza ${ }^{a, b, 14}$, F. Brivio ${ }^{a, b}$, V. Ciriolo, M.E. Dinardo ${ }^{a, b}$, S. Fiorendi ${ }^{a, b, 14}$, S. Gennai $^{a}$,

A. Ghezzi ${ }^{a, b}$, P. Govoni ${ }^{a, b}$, M. Malberti ${ }^{a}, b$, S. Malvezzi ${ }^{a}$, R.A. Manzoni ${ }^{a, b}$, D. Menasce ${ }^{a}$,

L. Moroni ${ }^{a}$, M. Paganoni ${ }^{a}, b$, D. Pedrini ${ }^{a}$, S. Pigazzini ${ }^{a, b}$, S. Ragazzi ${ }^{a, b}$, T. Tabarelli de Fatis $^{a, b}$

INFN Sezione di Napoli ${ }^{a}$, Università di Napoli 'Federico II' ${ }^{b}$, Napoli, Italy, Università della Basilicata ${ }^{c}$, Potenza, Italy, Università G. Marconi ${ }^{d}$, Roma, Italy

S. Buontempo ${ }^{a}$, N. Cavallo ${ }^{a, c}$, G. De Nardo ${ }^{a, b}$, S. Di Guida ${ }^{a, d, 14}$, F. Fabozzi ${ }^{a, c}$, F. Fienga ${ }^{a, b}$, A.O.M. Iorio ${ }^{a, b}$, L. Lista ${ }^{a}$, S. Meola ${ }^{a, d, 14}$, P. Paolucci ${ }^{a, 14}$, C. Sciacca ${ }^{a, b}$, F. Thyssen ${ }^{a}$

INFN Sezione di Padova ${ }^{a}$, Università di Padova ${ }^{b}$, Padova, Italy, Università di Trento ${ }^{c}$, Trento, Italy

P. Azzi ${ }^{a, 14}$, N. Bacchetta ${ }^{a}$, L. Benato ${ }^{a, b}$, D. Bisello ${ }^{a, b}$, A. Boletti ${ }^{a, b}$, R. Carlin ${ }^{a, b}$, A. Carvalho Antunes De Oliveira ${ }^{a, b}$, P. Checchia ${ }^{a}$, M. Dall'Osso ${ }^{a, b}$, P. De Castro Manzano ${ }^{a}$, T. Dorigo ${ }^{a}$, U. Dosselli ${ }^{a}$, F. Gasparini ${ }^{a, b}$, U. Gasparini ${ }^{a}, b$, A. Gozzelino ${ }^{a}$, S. Lacaprara ${ }^{a}$, M. Margoni ${ }^{a, b}$, A.T. Meneguzzo ${ }^{a, b}$, J. Pazzini ${ }^{a, b}$, N. Pozzobon ${ }^{a, b}$, P. Ronchese ${ }^{a, b}$,

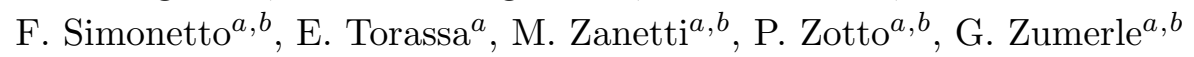

INFN Sezione di Pavia ${ }^{a}$, Università di Pavia ${ }^{b}$, Pavia, Italy

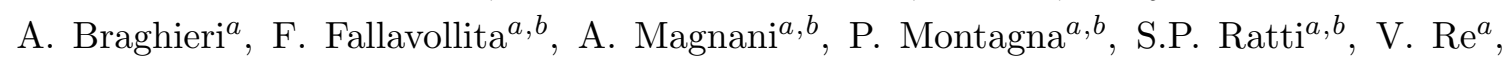
M. Ressegotti, C. Riccardi ${ }^{a, b}$, P. Salvini ${ }^{a}$, I. Vai ${ }^{a, b}$, P. Vitulo ${ }^{a, b}$

INFN Sezione di Perugia ${ }^{a}$, Università di Perugia ${ }^{b}$, Perugia, Italy

L. Alunni Solestizi ${ }^{a}, b$, G.M. Bilei ${ }^{a}$, D. Ciangottini ${ }^{a, b}$, L. Fanò ${ }^{a}, b$ P. Lariccia $^{a, b}$,

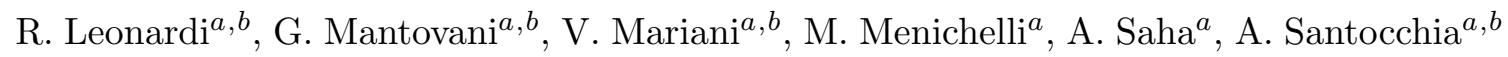

INFN Sezione di Pisa ${ }^{a}$, Università di Pisa ${ }^{b}$, Scuola Normale Superiore di Pisa ${ }^{c}$, Pisa, Italy

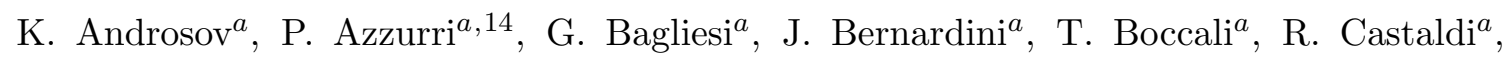

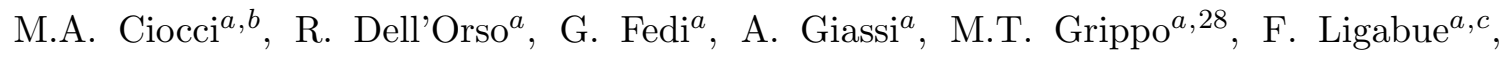




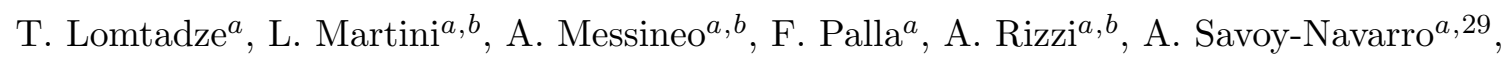

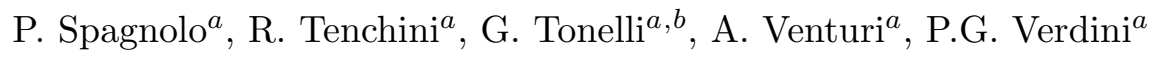

INFN Sezione di Roma ${ }^{a}$, Sapienza Università di Roma ${ }^{b}$, Rome, Italy

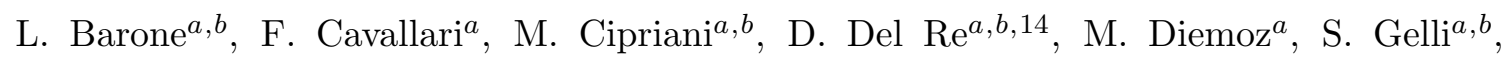
E. Longo ${ }^{a, b}$, F. Margaroli ${ }^{a, b}$, B. Marzocchi ${ }^{a, b}$, P. Meridiani ${ }^{a}$, G. Organtini ${ }^{a, b}$,

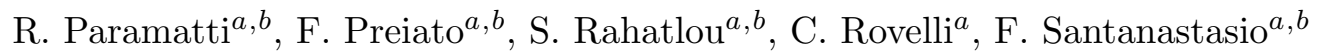

INFN Sezione di Torino ${ }^{a}$, Università di Torino ${ }^{b}$, Torino, Italy, Università del Piemonte Orientale ${ }^{c}$, Novara, Italy

N. Amapane ${ }^{a, b}$, R. Arcidiacono ${ }^{a, c, 14}$, S. Argiro ${ }^{a, b}$, M. Arneodo ${ }^{a, c}$, N. Bartosik ${ }^{a}$, R. Bellan ${ }^{a, b}$, C. Biino ${ }^{a}$, N. Cartiglia ${ }^{a}$, F. Cenna ${ }^{a, b}$, M. Costa ${ }^{a, b}$, R. Covarelli ${ }^{a, b}$, A. $\operatorname{Degano}^{a, b}$, N. Demaria ${ }^{a}$, B. Kiani ${ }^{a, b}$, C. Mariotti ${ }^{a}$, S. Masellia ${ }^{a}$, E. Migliore ${ }^{a, b}$, V. Monaco ${ }^{a, b}$, E. Monteil ${ }^{a, b}$, M. Monteno ${ }^{a}$, M.M. Obertino ${ }^{a}, b$, L. Pacher ${ }^{a}, b$, N. Pastrone ${ }^{a}$, M. Pelliccioni ${ }^{a}$, G.L. Pinna Angioni ${ }^{a, b}$, F. Ravera ${ }^{a, b}$, A. Romero ${ }^{a, b}$, M. Ruspa $^{a, c}$,

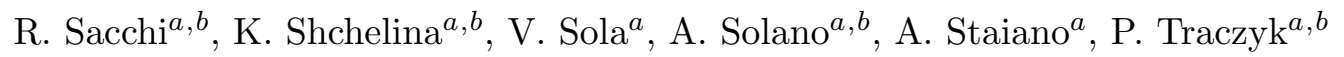

INFN Sezione di Trieste ${ }^{a}$, Università di Trieste ${ }^{b}$, Trieste, Italy

S. Belforte ${ }^{a}$, M. Casarsa ${ }^{a}$, F. Cossutti ${ }^{a}$, G. Della Ricca ${ }^{a, b}$, A. Zanetti $^{a}$

\section{Kyungpook National University, Daegu, Korea}

D.H. Kim, G.N. Kim, M.S. Kim, J. Lee, S. Lee, S.W. Lee, Y.D. Oh, S. Sekmen, D.C. Son, Y.C. Yang

Chonbuk National University, Jeonju, Korea

A. Lee

Chonnam National University, Institute for Universe and Elementary Particles, Kwangju, Korea

H. Kim

Hanyang University, Seoul, Korea

J.A. Brochero Cifuentes, J. Goh, T.J. Kim

Korea University, Seoul, Korea

S. Cho, S. Choi, Y. Go, D. Gyun, S. Ha, B. Hong, Y. Jo, Y. Kim, K. Lee, K.S. Lee, S. Lee, J. Lim, S.K. Park, Y. Roh

Seoul National University, Seoul, Korea

J. Almond, J. Kim, H. Lee, S.B. Oh, B.C. Radburn-Smith, S.h. Seo, U.K. Yang, H.D. Yoo, G.B. $\mathrm{Yu}$

University of Seoul, Seoul, Korea

M. Choi, H. Kim, J.H. Kim, J.S.H. Lee, I.C. Park, G. Ryu, M.S. Ryu

Sungkyunkwan University, Suwon, Korea

Y. Choi, C. Hwang, J. Lee, I. Yu

Vilnius University, Vilnius, Lithuania

V. Dudenas, A. Juodagalvis, J. Vaitkus 
National Centre for Particle Physics, Universiti Malaya, Kuala Lumpur, Malaysia

I. Ahmed, Z.A. Ibrahim, M.A.B. Md $\mathrm{Ali}^{30}$, F. Mohamad Idris ${ }^{31}$, W.A.T. Wan Abdullah, M.N. Yusli, Z. Zolkapli

Centro de Investigacion y de Estudios Avanzados del IPN, Mexico City, Mexico

H. Castilla-Valdez, E. De La Cruz-Burelo, I. Heredia-De La Cruz ${ }^{32}$, R. Lopez-Fernandez,

R. Magaña Villalba, J. Mejia Guisao, A. Sanchez-Hernandez

Universidad Iberoamericana, Mexico City, Mexico

S. Carrillo Moreno, C. Oropeza Barrera, F. Vazquez Valencia

Benemerita Universidad Autonoma de Puebla, Puebla, Mexico

S. Carpinteyro, I. Pedraza, H.A. Salazar Ibarguen, C. Uribe Estrada

Universidad Autónoma de San Luis Potosí, San Luis Potosí, Mexico

A. Morelos Pineda

University of Auckland, Auckland, New Zealand

D. Krofcheck

University of Canterbury, Christchurch, New Zealand

P.H. Butler

National Centre for Physics, Quaid-I-Azam University, Islamabad, Pakistan

A. Ahmad, M. Ahmad, Q. Hassan, H.R. Hoorani, W.A. Khan, A. Saddique, M.A. Shah, M. Shoaib, M. Waqas

National Centre for Nuclear Research, Swierk, Poland

H. Bialkowska, M. Bluj, B. Boimska, T. Frueboes, M. Górski, M. Kazana, K. Nawrocki,

K. Romanowska-Rybinska, M. Szleper, P. Zalewski

Institute of Experimental Physics, Faculty of Physics, University of Warsaw, Warsaw, Poland

K. Bunkowski, A. Byszuk ${ }^{33}$, K. Doroba, A. Kalinowski, M. Konecki, J. Krolikowski, M. Misiura, M. Olszewski, A. Pyskir, M. Walczak

Laboratório de Instrumentação e Física Experimental de Partículas, Lisboa, Portugal

P. Bargassa, C. Beirão Da Cruz E Silva, B. Calpas, A. Di Francesco, P. Faccioli, M. Gallinaro, J. Hollar, N. Leonardo, L. Lloret Iglesias, M.V. Nemallapudi, J. Seixas, O. Toldaiev, D. Vadruccio, J. Varela

Joint Institute for Nuclear Research, Dubna, Russia

S. Afanasiev, P. Bunin, M. Gavrilenko, I. Golutvin, I. Gorbunov, A. Kamenev, V. Karjavin, A. Lanev, A. Malakhov, V. Matveev ${ }^{34,35}$, V. Palichik, V. Perelygin, S. Shmatov, S. Shulha, N. Skatchkov, V. Smirnov, N. Voytishin, A. Zarubin 
Petersburg Nuclear Physics Institute, Gatchina (St. Petersburg), Russia

L. Chtchipounov, V. Golovtsov, Y. Ivanov, V. Kim ${ }^{36}$, E. Kuznetsova ${ }^{37}$, V. Murzin, V. Oreshkin, V. Sulimov, A. Vorobyev

Institute for Nuclear Research, Moscow, Russia

Yu. Andreev, A. Dermenev, S. Gninenko, N. Golubev, A. Karneyeu, M. Kirsanov, N. Krasnikov, A. Pashenkov, D. Tlisov, A. Toropin

Institute for Theoretical and Experimental Physics, Moscow, Russia

V. Epshteyn, V. Gavrilov, N. Lychkovskaya, V. Popov, I. Pozdnyakov, G. Safronov, A. Spiridonov, M. Toms, E. Vlasov, A. Zhokin

Moscow Institute of Physics and Technology, Moscow, Russia

T. Aushev, A. Bylinkin ${ }^{35}$

National Research Nuclear University 'Moscow Engineering Physics Institute' (MEPhI), Moscow, Russia

R. Chistov $^{38}$, M. Danilov ${ }^{38}$, E. Zhemchugov

P.N. Lebedev Physical Institute, Moscow, Russia

V. Andreev, M. Azarkin ${ }^{35}$, I. Dremin ${ }^{35}$, M. Kirakosyan, A. Leonidov ${ }^{35}$, A. Terkulov

Skobeltsyn Institute of Nuclear Physics, Lomonosov Moscow State University, Moscow, Russia

A. Baskakov, A. Belyaev, E. Boos, V. Bunichev, M. Dubinin ${ }^{39}$, L. Dudko, A. Ershov,

A. Gribushin, V. Klyukhin, O. Kodolova, I. Lokhtin, I. Miagkov, S. Obraztsov, M. Perfilov,

V. Savrin

Novosibirsk State University (NSU), Novosibirsk, Russia

V. Blinov ${ }^{40}$, Y.Skovpen $^{40}$, D. Shtol ${ }^{40}$

State Research Center of Russian Federation, Institute for High Energy Physics, Protvino, Russia

I. Azhgirey, I. Bayshev, S. Bitioukov, D. Elumakhov, V. Kachanov, A. Kalinin, D. Konstantinov, V. Krychkine, V. Petrov, R. Ryutin, A. Sobol, S. Troshin, N. Tyurin, A. Uzunian, A. Volkov

University of Belgrade, Faculty of Physics and Vinca Institute of Nuclear Sciences, Belgrade, Serbia

P. Adzic ${ }^{41}$, P. Cirkovic, D. Devetak, M. Dordevic, J. Milosevic, V. Rekovic

Centro de Investigaciones Energéticas Medioambientales y Tecnológicas (CIEMAT), Madrid, Spain

J. Alcaraz Maestre, M. Barrio Luna, E. Calvo, M. Cerrada, M. Chamizo Llatas, N. Colino, B. De La Cruz, A. Delgado Peris, A. Escalante Del Valle, C. Fernandez Bedoya, J.P. Fernández Ramos, J. Flix, M.C. Fouz, P. Garcia-Abia, O. Gonzalez Lopez, S. Goy Lopez, J.M. Hernandez, M.I. Josa, E. Navarro De Martino, A. Pérez-Calero Yzquierdo, J. Puerta Pelayo, A. Quintario Olmeda, I. Redondo, L. Romero, M.S. Soares 
Universidad Autónoma de Madrid, Madrid, Spain

J.F. de Trocóniz, M. Missiroli, D. Moran

Universidad de Oviedo, Oviedo, Spain

J. Cuevas, C. Erice, J. Fernandez Menendez, I. Gonzalez Caballero, J.R. González Fernández, E. Palencia Cortezon, S. Sanchez Cruz, I. Suárez Andrés, P. Vischia, J.M. Vizan Garcia

Instituto de Física de Cantabria (IFCA), CSIC-Universidad de Cantabria, Santander, Spain

I.J. Cabrillo, A. Calderon, E. Curras, M. Fernandez, J. Garcia-Ferrero, G. Gomez, A. Lopez Virto, J. Marco, C. Martinez Rivero, F. Matorras, J. Piedra Gomez, T. Rodrigo, A. RuizJimeno, L. Scodellaro, N. Trevisani, I. Vila, R. Vilar Cortabitarte

CERN, European Organization for Nuclear Research, Geneva, Switzerland

D. Abbaneo, E. Auffray, G. Auzinger, P. Baillon, A.H. Ball, D. Barney, P. Bloch, A. Bocci,

C. Botta, T. Camporesi, R. Castello, M. Cepeda, G. Cerminara, Y. Chen, A. Cimmino, D. d'Enterria, A. Dabrowski, V. Daponte, A. David, M. De Gruttola, A. De Roeck, E. Di Marco $^{42}$, M. Dobson, B. Dorney, T. du Pree, M. Dünser, N. Dupont, A. Elliott-Peisert, P. Everaerts, S. Fartoukh, G. Franzoni, J. Fulcher, W. Funk, D. Gigi, K. Gill, M. Girone, F. Glege, D. Gulhan, S. Gundacker, M. Guthoff, P. Harris, J. Hegeman, V. Innocente, P. Janot, J. Kieseler, H. Kirschenmann, V. Knünz, A. Kornmayer ${ }^{14}$, M.J. Kortelainen, M. Krammer ${ }^{1}$, C. Lange, P. Lecoq, C. Lourenço, M.T. Lucchini, L. Malgeri, M. Mannelli, A. Martelli, F. Meijers, J.A. Merlin, S. Mersi, E. Meschi, P. Milenovic ${ }^{43}$, F. Moortgat, S. Morovic, M. Mulders, H. Neugebauer, S. Orfanelli, L. Orsini, L. Pape, E. Perez, M. Peruzzi, A. Petrilli, G. Petrucciani, A. Pfeiffer, M. Pierini, A. Racz, T. Reis, G. Rolandi ${ }^{44}$, M. Rovere, H. Sakulin, J.B. Sauvan, C. Schäfer, C. Schwick, M. Seidel, M. Selvaggi, A. Sharma, P. Silva, P. Sphicas ${ }^{45}$, J. Steggemann, M. Stoye, Y. Takahashi, M. Tosi, D. Treille, A. Triossi, A. Tsirou, V. Veckalns ${ }^{46}$, G.I. Veres ${ }^{19}$, M. Verweij, N. Wardle, H.K. Wöhri, A. Zagozdzinska ${ }^{33}$, W.D. Zeuner

Paul Scherrer Institut, Villigen, Switzerland

W. Bertl, K. Deiters, W. Erdmann, R. Horisberger, Q. Ingram, H.C. Kaestli, D. Kotlinski, U. Langenegger, T. Rohe, S.A. Wiederkehr

Institute for Particle Physics, ETH Zurich, Zurich, Switzerland

F. Bachmair, L. Bäni, L. Bianchini, B. Casal, G. Dissertori, M. Dittmar, M. Donegà, C. Grab, C. Heidegger, D. Hits, J. Hoss, G. Kasieczka, W. Lustermann, B. Mangano, M. Marionneau, P. Martinez Ruiz del Arbol, M. Masciovecchio, M.T. Meinhard, D. Meister, F. Micheli, P. Musella, F. Nessi-Tedaldi, F. Pandolfi, J. Pata, F. Pauss, G. Perrin, L. Perrozzi, M. Quittnat, M. Rossini, M. Schönenberger, A. Starodumov ${ }^{47}$, V.R. Tavolaro, K. Theofilatos, R. Wallny

Universität Zürich, Zurich, Switzerland

T.K. Aarrestad, C. Amsler ${ }^{48}$, L. Caminada, M.F. Canelli, A. De Cosa, S. Donato, C. Galloni, A. Hinzmann, T. Hreus, B. Kilminster, J. Ngadiuba, D. Pinna, G. Rauco, 
P. Robmann, D. Salerno, C. Seitz, Y. Yang, A. Zucchetta

National Central University, Chung-Li, Taiwan

V. Candelise, T.H. Doan, Sh. Jain, R. Khurana, M. Konyushikhin, C.M. Kuo, W. Lin, A. Pozdnyakov, S.S. Yu

National Taiwan University (NTU), Taipei, Taiwan

Arun Kumar, P. Chang, Y.H. Chang, Y. Chao, K.F. Chen, P.H. Chen, F. Fiori, W.-S. Hou, Y. Hsiung, Y.F. Liu, R.-S. Lu, M. Miñano Moya, E. Paganis, A. Psallidas, J.f. Tsai

Chulalongkorn University, Faculty of Science, Department of Physics, Bangkok, Thailand

B. Asavapibhop, G. Singh, N. Srimanobhas, N. Suwonjandee

Cukurova University, Physics Department, Science and Art Faculty, Adana, Turkey

A. Adiguzel, M.N. Bakirci ${ }^{49}$, F. Boran, S. Damarseckin, Z.S. Demiroglu, C. Dozen, E. Eskut, S. Girgis, G. Gokbulut, Y. Guler, I. $\mathrm{Hos}^{50}$, E.E. Kangal ${ }^{51}$, O. Kara, U. Kiminsu, M. Oglakci, G. Onengut ${ }^{52}$, K. Ozdemir ${ }^{53}$, S. Ozturk ${ }^{49}$, A. Polatoz, D. Sunar Cerci ${ }^{54}$, S. Turkcapar, I.S. Zorbakir, C. Zorbilmez

Middle East Technical University, Physics Department, Ankara, Turkey

B. Bilin, B. Isildak ${ }^{55}$, G. Karapinar ${ }^{56}$, M. Yalvac, M. Zeyrek

Bogazici University, Istanbul, Turkey

E. Gülmez, M. Kaya ${ }^{57}$, O. Kaya ${ }^{58}$, E.A. Yetkin ${ }^{59}$, T. Yetkin ${ }^{60}$

Istanbul Technical University, Istanbul, Turkey

A. Cakir, K. Cankocak, S. Sen ${ }^{61}$

Institute for Scintillation Materials of National Academy of Science of Ukraine, Kharkov, Ukraine

B. Grynyov

National Scientific Center, Kharkov Institute of Physics and Technology, Kharkov, Ukraine

L. Levchuk, P. Sorokin

University of Bristol, Bristol, United Kingdom

R. Aggleton, F. Ball, L. Beck, J.J. Brooke, D. Burns, E. Clement, D. Cussans, H. Flacher,

J. Goldstein, M. Grimes, G.P. Heath, H.F. Heath, J. Jacob, L. Kreczko, C. Lucas, D.M. Newbold ${ }^{6}$, S. Paramesvaran, A. Poll, T. Sakuma, S. Seif El Nasr-storey, D. Smith, V.J. Smith

Rutherford Appleton Laboratory, Didcot, United Kingdom

K.W. Bell, A. Belyaev ${ }^{63}$, C. Brew, R.M. Brown, L. Calligaris, D. Cieri, D.J.A. Cockerill, J.A. Coughlan, K. Harder, S. Harper, E. Olaiya, D. Petyt, C.H. Shepherd-Themistocleous, A. Thea, I.R. Tomalin, T. Williams 


\section{Imperial College, London, United Kingdom}

M. Baber, R. Bainbridge, O. Buchmuller, A. Bundock, S. Casasso, M. Citron, D. Colling, L. Corpe, P. Dauncey, G. Davies, A. De Wit, M. Della Negra, R. Di Maria, P. Dunne, A. Elwood, D. Futyan, Y. Haddad, G. Hall, G. Iles, T. James, R. Lane, C. Laner, L. Lyons, A.-M. Magnan, S. Malik, L. Mastrolorenzo, J. Nash, A. Nikitenko ${ }^{47}$, J. Pela, B. Penning, M. Pesaresi, D.M. Raymond, A. Richards, A. Rose, E. Scott, C. Seez, S. Summers, A. Tapper, K. Uchida, M. Vazquez Acosta ${ }^{64}$, T. Virdee ${ }^{14}$, J. Wright, S.C. Zenz

\section{Brunel University, Uxbridge, United Kingdom}

J.E. Cole, P.R. Hobson, A. Khan, P. Kyberd, I.D. Reid, P. Symonds, L. Teodorescu, M. Turner

\section{Baylor University, Waco, U.S.A.}

A. Borzou, K. Call, J. Dittmann, K. Hatakeyama, H. Liu, N. Pastika

\section{Catholic University of America, Washington, U.S.A.}

R. Bartek, A. Dominguez

The University of Alabama, Tuscaloosa, U.S.A.

A. Buccilli, S.I. Cooper, C. Henderson, P. Rumerio, C. West

Boston University, Boston, U.S.A.

D. Arcaro, A. Avetisyan, T. Bose, D. Gastler, D. Rankin, C. Richardson, J. Rohlf, L. Sulak,

D. Zou

\section{Brown University, Providence, U.S.A.}

G. Benelli, D. Cutts, A. Garabedian, J. Hakala, U. Heintz, J.M. Hogan, O. Jesus, K.H.M. Kwok, E. Laird, G. Landsberg, Z. Mao, M. Narain, S. Piperov, S. Sagir, E. Spencer, R. Syarif

\section{University of California, Davis, Davis, U.S.A.}

R. Breedon, D. Burns, M. Calderon De La Barca Sanchez, S. Chauhan, M. Chertok, J. Conway, R. Conway, P.T. Cox, R. Erbacher, C. Flores, G. Funk, M. Gardner, W. Ko, R. Lander, C. Mclean, M. Mulhearn, D. Pellett, J. Pilot, S. Shalhout, M. Shi, J. Smith, M. Squires, D. Stolp, K. Tos, M. Tripathi

University of California, Los Angeles, U.S.A.

M. Bachtis, C. Bravo, R. Cousins, A. Dasgupta, A. Florent, J. Hauser, M. Ignatenko, N. Mccoll, D. Saltzberg, C. Schnaible, V. Valuev, M. Weber

University of California, Riverside, Riverside, U.S.A.

E. Bouvier, K. Burt, R. Clare, J. Ellison, J.W. Gary, S.M.A. Ghiasi Shirazi, G. Hanson, J. Heilman, P. Jandir, E. Kennedy, F. Lacroix, O.R. Long, M. Olmedo Negrete, M.I. Paneva, A. Shrinivas, W. Si, H. Wei, S. Wimpenny, B. R. Yates

\section{University of California, San Diego, La Jolla, U.S.A.}

J.G. Branson, G.B. Cerati, S. Cittolin, M. Derdzinski, R. Gerosa, A. Holzner, D. Klein, V. Krutelyov, J. Letts, I. Macneill, D. Olivito, S. Padhi, M. Pieri, M. Sani, V. Sharma, 
S. Simon, M. Tadel, A. Vartak, S. Wasserbaech ${ }^{65}$, C. Welke, J. Wood, F. Würthwein, A. Yagil, G. Zevi Della Porta

University of California, Santa Barbara - Department of Physics, Santa Barbara, U.S.A.

N. Amin, R. Bhandari, J. Bradmiller-Feld, C. Campagnari, A. Dishaw, V. Dutta, M. Franco Sevilla, C. George, F. Golf, L. Gouskos, J. Gran, R. Heller, J. Incandela, S.D. Mullin, A. Ovcharova, H. Qu, J. Richman, D. Stuart, I. Suarez, J. Yoo

California Institute of Technology, Pasadena, U.S.A.

D. Anderson, J. Bendavid, A. Bornheim, J. Bunn, J.M. Lawhorn, A. Mott, H.B. Newman,

C. Pena, M. Spiropulu, J.R. Vlimant, S. Xie, R.Y. Zhu

Carnegie Mellon University, Pittsburgh, U.S.A.

M.B. Andrews, T. Ferguson, M. Paulini, J. Russ, M. Sun, H. Vogel, I. Vorobiev, M. Weinberg

University of Colorado Boulder, Boulder, U.S.A.

J.P. Cumalat, W.T. Ford, F. Jensen, A. Johnson, M. Krohn, S. Leontsinis, T. Mulholland, K. Stenson, S.R. Wagner

Cornell University, Ithaca, U.S.A.

J. Alexander, J. Chaves, J. Chu, S. Dittmer, K. Mcdermott, N. Mirman, J.R. Patterson, A. Rinkevicius, A. Ryd, L. Skinnari, L. Soffi, S.M. Tan, Z. Tao, J. Thom, J. Tucker, P. Wittich, M. Zientek

Fairfield University, Fairfield, U.S.A.

D. Winn

Fermi National Accelerator Laboratory, Batavia, U.S.A.

S. Abdullin, M. Albrow, G. Apollinari, A. Apresyan, S. Banerjee, L.A.T. Bauerdick, A. Beretvas, J. Berryhill, P.C. Bhat, G. Bolla, K. Burkett, J.N. Butler, H.W.K. Cheung, F. Chlebana, S. Cihangir ${ }^{\dagger}$, M. Cremonesi, J. Duarte, V.D. Elvira, I. Fisk, J. Freeman, E. Gottschalk, L. Gray, D. Green, S. Grünendahl, O. Gutsche, R.M. Harris, S. Hasegawa, J. Hirschauer, Z. Hu, B. Jayatilaka, S. Jindariani, M. Johnson, U. Joshi, B. Klima, B. Kreis, S. Lammel, J. Linacre, D. Lincoln, R. Lipton, M. Liu, T. Liu, R. Lopes De Sá, J. Lykken, K. Maeshima, N. Magini, J.M. Marraffino, S. Maruyama, D. Mason, P. McBride, P. Merkel, S. Mrenna, S. Nahn, V. O’Dell, K. Pedro, O. Prokofyev, G. Rakness, L. Ristori, E. SextonKennedy, A. Soha, W.J. Spalding, L. Spiegel, S. Stoynev, J. Strait, N. Strobbe, L. Taylor, S. Tkaczyk, N.V. Tran, L. Uplegger, E.W. Vaandering, C. Vernieri, M. Verzocchi, R. Vidal, M. Wang, H.A. Weber, A. Whitbeck, Y. Wu

University of Florida, Gainesville, U.S.A.

D. Acosta, P. Avery, P. Bortignon, D. Bourilkov, A. Brinkerhoff, A. Carnes, M. Carver, D. Curry, S. Das, R.D. Field, I.K. Furic, J. Konigsberg, A. Korytov, J.F. Low, P. Ma, K. Matchev, H. Mei, G. Mitselmakher, D. Rank, L. Shchutska, D. Sperka, L. Thomas, J. Wang, S. Wang, J. Yelton 
Florida International University, Miami, U.S.A.

S. Linn, P. Markowitz, G. Martinez, J.L. Rodriguez

Florida State University, Tallahassee, U.S.A.

A. Ackert, T. Adams, A. Askew, S. Bein, S. Hagopian, V. Hagopian, K.F. Johnson,

T. Kolberg, T. Perry, H. Prosper, A. Santra, R. Yohay

Florida Institute of Technology, Melbourne, U.S.A.

M.M. Baarmand, V. Bhopatkar, S. Colafranceschi, M. Hohlmann, D. Noonan, T. Roy, F. Yumiceva

University of Illinois at Chicago (UIC), Chicago, U.S.A.

M.R. Adams, L. Apanasevich, D. Berry, R.R. Betts, R. Cavanaugh, X. Chen, O. Evdokimov, C.E. Gerber, D.A. Hangal, D.J. Hofman, K. Jung, J. Kamin, I.D. Sandoval Gonzalez, H. Trauger, N. Varelas, H. Wang, Z. Wu, J. Zhang

The University of Iowa, Iowa City, U.S.A.

B. Bilki ${ }^{66}$, W. Clarida, K. Dilsiz, S. Durgut, R.P. Gandrajula, M. Haytmyradov, V. Khristenko, J.-P. Merlo, H. Mermerkaya ${ }^{67}$, A. Mestvirishvili, A. Moeller, J. Nachtman, H. Ogul, Y. Onel, F. Ozok ${ }^{68}$, A. Penzo, C. Snyder, E. Tiras, J. Wetzel, K. Yi

Johns Hopkins University, Baltimore, U.S.A.

B. Blumenfeld, A. Cocoros, N. Eminizer, D. Fehling, L. Feng, A.V. Gritsan, P. Maksimovic, J. Roskes, U. Sarica, M. Swartz, M. Xiao, C. You

The University of Kansas, Lawrence, U.S.A.

A. Al-bataineh, P. Baringer, A. Bean, S. Boren, J. Bowen, J. Castle, L. Forthomme, S. Khalil, A. Kropivnitskaya, D. Majumder, W. Mcbrayer, M. Murray, S. Sanders, R. Stringer, J.D. Tapia Takaki, Q. Wang

Kansas State University, Manhattan, U.S.A.

A. Ivanov, K. Kaadze, Y. Maravin, A. Mohammadi, L.K. Saini, N. Skhirtladze, S. Toda

Lawrence Livermore National Laboratory, Livermore, U.S.A.

F. Rebassoo, D. Wright

University of Maryland, College Park, U.S.A.

C. Anelli, A. Baden, O. Baron, A. Belloni, B. Calvert, S.C. Eno, C. Ferraioli, N.J. Hadley, S. Jabeen, G.Y. Jeng, R.G. Kellogg, J. Kunkle, A.C. Mignerey, F. Ricci-Tam, Y.H. Shin, A. Skuja, M.B. Tonjes, S.C. Tonwar

Massachusetts Institute of Technology, Cambridge, U.S.A.

D. Abercrombie, B. Allen, A. Apyan, V. Azzolini, R. Barbieri, A. Baty, R. Bi, K. Bierwagen, S. Brandt, W. Busza, I.A. Cali, M. D'Alfonso, Z. Demiragli, G. Gomez Ceballos, M. Goncharov, D. Hsu, Y. Iiyama, G.M. Innocenti, M. Klute, D. Kovalskyi, K. Krajczar, Y.S. Lai, Y.-J. Lee, A. Levin, P.D. Luckey, B. Maier, A.C. Marini, C. Mcginn, C. Mironov, S. Narayanan, X. Niu, C. Paus, C. Roland, G. Roland, J. Salfeld-Nebgen, G.S.F. Stephans, K. Tatar, D. Velicanu, J. Wang, T.W. Wang, B. Wyslouch 
University of Minnesota, Minneapolis, U.S.A.

A.C. Benvenuti, R.M. Chatterjee, A. Evans, P. Hansen, S. Kalafut, S.C. Kao, Y. Kubota, Z. Lesko, J. Mans, S. Nourbakhsh, N. Ruckstuhl, R. Rusack, N. Tambe, J. Turkewitz

University of Mississippi, Oxford, U.S.A.

J.G. Acosta, S. Oliveros

University of Nebraska-Lincoln, Lincoln, U.S.A.

E. Avdeeva, K. Bloom, D.R. Claes, C. Fangmeier, R. Gonzalez Suarez, R. Kamalieddin, I. Kravchenko, A. Malta Rodrigues, J. Monroy, J.E. Siado, G.R. Snow, B. Stieger

State University of New York at Buffalo, Buffalo, U.S.A.

M. Alyari, J. Dolen, A. Godshalk, C. Harrington, I. Iashvili, D. Nguyen, A. Parker, S. Rappoccio, B. Roozbahani

Northeastern University, Boston, U.S.A.

G. Alverson, E. Barberis, A. Hortiangtham, A. Massironi, D.M. Morse, D. Nash, T. Orimoto, R. Teixeira De Lima, D. Trocino, R.-J. Wang, D. Wood

Northwestern University, Evanston, U.S.A.

S. Bhattacharya, O. Charaf, K.A. Hahn, N. Mucia, N. Odell, B. Pollack, M.H. Schmitt, K. Sung, M. Trovato, M. Velasco

University of Notre Dame, Notre Dame, U.S.A.

N. Dev, M. Hildreth, K. Hurtado Anampa, C. Jessop, D.J. Karmgard, N. Kellams, K. Lannon, N. Marinelli, F. Meng, C. Mueller, Y. Musienko ${ }^{34}$, M. Planer, A. Reinsvold, R. Ruchti, N. Rupprecht, G. Smith, S. Taroni, M. Wayne, M. Wolf, A. Woodard

The Ohio State University, Columbus, U.S.A.

J. Alimena, L. Antonelli, B. Bylsma, L.S. Durkin, S. Flowers, B. Francis, A. Hart, C. Hill, W. Ji, B. Liu, W. Luo, D. Puigh, B.L. Winer, H.W. Wulsin

Princeton University, Princeton, U.S.A.

S. Cooperstein, O. Driga, P. Elmer, J. Hardenbrook, P. Hebda, D. Lange, J. Luo, D. Marlow, T. Medvedeva, K. Mei, I. Ojalvo, J. Olsen, C. Palmer, P. Piroué, D. Stickland, A. Svyatkovskiy, C. Tully

University of Puerto Rico, Mayaguez, U.S.A.

S. Malik

Purdue University, West Lafayette, U.S.A.

A. Barker, V.E. Barnes, S. Folgueras, L. Gutay, M.K. Jha, M. Jones, A.W. Jung, A. Khatiwada, D.H. Miller, N. Neumeister, J.F. Schulte, J. Sun, F. Wang, W. Xie

Purdue University Northwest, Hammond, U.S.A.

N. Parashar, J. Stupak

Rice University, Houston, U.S.A.

A. Adair, B. Akgun, Z. Chen, K.M. Ecklund, F.J.M. Geurts, M. Guilbaud, W. Li, B. Michlin, M. Northup, B.P. Padley, J. Roberts, J. Rorie, Z. Tu, J. Zabel 
University of Rochester, Rochester, U.S.A.

B. Betchart, A. Bodek, P. de Barbaro, R. Demina, Y.t. Duh, T. Ferbel, M. Galanti, A. Garcia-Bellido, J. Han, O. Hindrichs, A. Khukhunaishvili, K.H. Lo, P. Tan, M. Verzetti Rutgers, The State University of New Jersey, Piscataway, U.S.A.

A. Agapitos, J.P. Chou, Y. Gershtein, T.A. Gómez Espinosa, E. Halkiadakis, M. Heindl, E. Hughes, S. Kaplan, R. Kunnawalkam Elayavalli, S. Kyriacou, A. Lath, R. Montalvo, K. Nash, M. Osherson, H. Saka, S. Salur, S. Schnetzer, D. Sheffield, S. Somalwar, R. Stone, S. Thomas, P. Thomassen, M. Walker

University of Tennessee, Knoxville, U.S.A.

A.G. Delannoy, M. Foerster, J. Heideman, G. Riley, K. Rose, S. Spanier, K. Thapa

Texas A\&M University, College Station, U.S.A.

O. Bouhali ${ }^{69}$, A. Celik, M. Dalchenko, M. De Mattia, A. Delgado, S. Dildick, R. Eusebi, J. Gilmore, T. Huang, E. Juska, T. Kamon ${ }^{70}$, R. Mueller, Y. Pakhotin, R. Patel, A. Perloff, L. Perniè, D. Rathjens, A. Safonov, A. Tatarinov, K.A. Ulmer

Texas Tech University, Lubbock, U.S.A.

N. Akchurin, J. Damgov, F. De Guio, C. Dragoiu, P.R. Dudero, J. Faulkner, E. Gurpinar, S. Kunori, K. Lamichhane, S.W. Lee, T. Libeiro, T. Peltola, S. Undleeb, I. Volobouev, Z. Wang

Vanderbilt University, Nashville, U.S.A.

S. Greene, A. Gurrola, R. Janjam, W. Johns, C. Maguire, A. Melo, H. Ni, P. Sheldon, S. Tuo, J. Velkovska, Q. Xu

University of Virginia, Charlottesville, U.S.A.

M.W. Arenton, P. Barria, B. Cox, R. Hirosky, A. Ledovskoy, H. Li, C. Neu, T. Sinthuprasith, X. Sun, Y. Wang, E. Wolfe, F. Xia

Wayne State University, Detroit, U.S.A.

C. Clarke, R. Harr, P.E. Karchin, J. Sturdy, S. Zaleski

University of Wisconsin - Madison, Madison, WI, U.S.A.

D.A. Belknap, J. Buchanan, C. Caillol, S. Dasu, L. Dodd, S. Duric, B. Gomber, M. Grothe, M. Herndon, A. Hervé, U. Hussain, P. Klabbers, A. Lanaro, A. Levine, K. Long, R. Loveless, G.A. Pierro, G. Polese, T. Ruggles, A. Savin, N. Smith, W.H. Smith, D. Taylor, N. Woods

$\dagger$ : Deceased

1: Also at Vienna University of Technology, Vienna, Austria

2: Also at State Key Laboratory of Nuclear Physics and Technology, Peking University, Beijing, China

3: Also at Universidade Estadual de Campinas, Campinas, Brazil

4: Also at Universidade Federal de Pelotas, Pelotas, Brazil

5: Also at Université Libre de Bruxelles, Bruxelles, Belgium

6: Also at Universidad de Antioquia, Medellin, Colombia 
7: Also at Joint Institute for Nuclear Research, Dubna, Russia

8: Also at Suez University, Suez, Egypt

9: Now at British University in Egypt, Cairo, Egypt

10: Also at Fayoum University, El-Fayoum, Egypt

11: Now at Helwan University, Cairo, Egypt

12: Also at Université de Haute Alsace, Mulhouse, France

13: Also at Skobeltsyn Institute of Nuclear Physics, Lomonosov Moscow State University, Moscow, Russia

14: Also at CERN, European Organization for Nuclear Research, Geneva, Switzerland

15: Also at RWTH Aachen University, III. Physikalisches Institut A, Aachen, Germany

16: Also at University of Hamburg, Hamburg, Germany

17: Also at Brandenburg University of Technology, Cottbus, Germany

18: Also at Institute of Nuclear Research ATOMKI, Debrecen, Hungary

19: Also at MTA-ELTE Lendület CMS Particle and Nuclear Physics Group, Eötvös Loránd University, Budapest, Hungary

20: Also at Institute of Physics, University of Debrecen, Debrecen, Hungary

21: Also at Indian Institute of Technology Bhubaneswar, Bhubaneswar, India

22: Also at University of Visva-Bharati, Santiniketan, India

23: Also at Institute of Physics, Bhubaneswar, India

24: Also at University of Ruhuna, Matara, Sri Lanka

25: Also at Isfahan University of Technology, Isfahan, Iran

26: Also at Yazd University, Yazd, Iran

27: Also at Plasma Physics Research Center, Science and Research Branch, Islamic Azad University, Tehran, Iran

28: Also at Università degli Studi di Siena, Siena, Italy

29: Also at Purdue University, West Lafayette, U.S.A.

30: Also at International Islamic University of Malaysia, Kuala Lumpur, Malaysia

31: Also at Malaysian Nuclear Agency, MOSTI, Kajang, Malaysia

32: Also at Consejo Nacional de Ciencia y Tecnología, Mexico city, Mexico

33: Also at Warsaw University of Technology, Institute of Electronic Systems, Warsaw, Poland

34: Also at Institute for Nuclear Research, Moscow, Russia

35: Now at National Research Nuclear University 'Moscow Engineering Physics Institute' (MEPhI), Moscow, Russia

36: Also at St. Petersburg State Polytechnical University, St. Petersburg, Russia

37: Also at University of Florida, Gainesville, U.S.A.

38: Also at P.N. Lebedev Physical Institute, Moscow, Russia

39: Also at California Institute of Technology, Pasadena, U.S.A.

40: Also at Budker Institute of Nuclear Physics, Novosibirsk, Russia

41: Also at Faculty of Physics, University of Belgrade, Belgrade, Serbia

42: Also at INFN Sezione di Roma; Sapienza Università di Roma, Rome, Italy

43: Also at University of Belgrade, Faculty of Physics and Vinca Institute of Nuclear Sciences, Belgrade, Serbia

44: Also at Scuola Normale e Sezione dell'INFN, Pisa, Italy

45: Also at National and Kapodistrian University of Athens, Athens, Greece

46: Also at Riga Technical University, Riga, Latvia

47: Also at Institute for Theoretical and Experimental Physics, Moscow, Russia

48: Also at Albert Einstein Center for Fundamental Physics, Bern, Switzerland

49: Also at Gaziosmanpasa University, Tokat, Turkey 
50: Also at Istanbul Aydin University, Istanbul, Turkey

51: Also at Mersin University, Mersin, Turkey

52: Also at Cag University, Mersin, Turkey

53: Also at Piri Reis University, Istanbul, Turkey

54: Also at Adiyaman University, Adiyaman, Turkey

55: Also at Ozyegin University, Istanbul, Turkey

56: Also at Izmir Institute of Technology, Izmir, Turkey

57: Also at Marmara University, Istanbul, Turkey

58: Also at Kafkas University, Kars, Turkey

59: Also at Istanbul Bilgi University, Istanbul, Turkey

60: Also at Yildiz Technical University, Istanbul, Turkey

61: Also at Hacettepe University, Ankara, Turkey

62: Also at Rutherford Appleton Laboratory, Didcot, United Kingdom

63: Also at School of Physics and Astronomy, University of Southampton, Southampton, United Kingdom

64: Also at Instituto de Astrofísica de Canarias, La Laguna, Spain

65: Also at Utah Valley University, Orem, U.S.A.

66: Also at BEYKENT UNIVERSITY, Istanbul, Turkey

67: Also at Erzincan University, Erzincan, Turkey

68: Also at Mimar Sinan University, Istanbul, Istanbul, Turkey

69: Also at Texas A\&M University at Qatar, Doha, Qatar

70: Also at Kyungpook National University, Daegu, Korea 